\title{
On the Numerical Dispersion of Electromagnetic Particle-In-Cell Code : Finite Grid Instability
}

\author{
M. D. Meyers ${ }^{* 1,2}$, C.-K. Huang ${ }^{\dagger 1}$, Y. Zeng ${ }^{\ddagger 1}$, S. A. Yi ${ }^{1}$, and B. J. \\ Albright ${ }^{1}$ \\ ${ }^{1}$ Los Alamos National Laboratory, Los Alamos, New Mexico 87545, USA \\ ${ }^{2}$ Department of Physics and Astronomy, University of California Los \\ Angeles, Los Angeles, California 90095, USA
}

May 11, 2015

Keywords: Particle-In-Cell, Plasma Simulation, Relativistic Plasma Drift, Numerical Dispersion Relation, Numerical Instability, Finite Grid Instability.

\begin{abstract}
The Particle-In-Cell (PIC) method is widely used in relativistic particle beam and laser plasma modeling. However, the PIC method exhibits numerical instabilities that can render unphysical simulation results or even destroy the simulation. For electromagnetic relativistic beam and plasma modeling, the most relevant numerical instabilities are the finite grid instability and the numerical Cherenkov instability. We review the numerical dispersion relation of the Electromagnetic PIC model. We rigorously derive the faithful 3-D numerical dispersion relation of the PIC model, for a simple, direct current deposition scheme, which does not conserve electric charge exactly. We then specialize to the Yee FDTD scheme. In particular, we clarify the presence of alias modes in an eigenmode analysis of the PIC model, which combines both discrete and continuous variables. The manner in which the PIC model updates and samples the fields and distribution function, together with the temporal and spatial phase factors from solving Maxwell's equations on the Yee grid with the leapfrog scheme, are explicitly accounted for. Numerical solutions to the electrostatic-like modes in the 1-D dispersion relation for a cold drifting plasma are obtained for parameters of interest. In the succeeding analysis, we investigate how the finite grid instability arises from the interaction of the numerical modes admitted in the system and their aliases. The most significant interaction is due critically to the correct representation of the operators in the dispersion relation.
\end{abstract}

*mdmeyers@physics.ucla.edu

†huangck@lanl.gov, corresponding author

${ }^{\ddagger}$ current address: Halliburton Energy Services, Houston, Texas 77032, USA 
We obtain a simple analytic expression for the peak growth rate due to this interaction, which is then verified by simulation. We demonstrate that our analysis is readily extendable to charge conserving models.

\section{Introduction}

The Particle-In-Cell (PIC) method $[1,2,3]$ is widely used in numerical simulations of the kinetic behavior of plasmas and particle beams. The PIC model is a combination of the Lagrangian model for the particles, in which particles can freely move in the simulation domain (subject to boundary conditions) and the grid-based model for the electromagnetic fields, where the fields are solved on the grid from the charge and current of the particles evaluated onto the grid. Contrary to the difference between the particles' continuous spatial variable and the fields' discrete spatial variable (which requires gather/scatter operations in each simulation step), all particle and field quantities in the PIC model are defined on a discrete time variable. (Albeit with necessary constant time offsets between these quantities due to the staggering of the temporal grid. Similarly, there is spatial staggering.) As a result, no equivalent gather/scatter operation in time is necessary. A typical Electromagnetic PIC code (referred to as the standard E.M. PIC hereafter) adopts the Yee [4] Finite Difference Time Domain (FDTD) scheme for solving Maxwell's equations. The Yee FDTD scheme (and other similar FDTD schemes) by itself, when applied to a simulation of a simple, linear medium, is stable as long as the Courant condition is satisfied. However, its coupling with the continuous Lagrangian particle model in PIC is susceptible to various numerical instabilities - some are encumbering but generally benign, such as grid heating [5], but many others are very virulent. Recent examples of the latter are the numerical instabilities found in a relativistically drifting cold plasma for the simulation of the laser wakefield accelerator [6] and collisionless shock [7,8]. Numerical instability also inflates the inherent noise level in a realistic PIC simulation, potentially leading to "spurious physics," i.e., unphysical numerical artifacts.

The occurrence of numerical instabilities in PIC usually depends not only on the numerical parameters, like the grid size and time step size, but also on the detailed particle phase space distribution through each step in the PIC computation loop. For example, a PIC simulation with a cold particle distribution can exhibit numerical instabilities with different characteristics than those in a simulation with a Maxwellian particle distribution. Clearly, for the stability analysis of such a "numerical plasma," an accurate numerical analysis based on the actual PIC model, not some approximate system, is desirable.

Similar to the analysis of other dynamical systems, one way to study the numerical stability of the PIC model is to investigate the intrinsic modes that the system supports. Such an eigenmode analysis is done by assuming a single monochromatic perturbation (usually in the form of a plane wave for a system with translational symmetry) already exists on some parts/quantities in the system, and then investigating its impact on other parts/quantities of the system, and finally the feedback onto the perturbation itself. It is worth noting that the perturbation should be on the internal variables of the isolated 
system under investigation, not on some external variables that are connected to the corresponding internal variables, e.g., applying an external field on the system or sampling a continuous perturbation into the discrete variables of the system. Furthermore, the perturbation is considered to be smooth without discrete particle noise (i.e., in the limit the number of particles per cell $N \rightarrow \infty$ ) and to have been applied for an infinite time, so that, in principle, this is not an initial value problem.

Mathematically, the above analysis results in an equation describing how a perturbation on some part of the system propagates to other parts, and eventually feeds back to itself. For a nonlinear system, this is typically very complicated and one often linearizes the response of each part of the system so as to study the linear properties of the system. We note that this linear approximation, justified by assuming the perturbation is small, is necessary to study the eigenmodes of the linear system and should not be confused with other approximations made in order to simplify the algebra. These are additional approximations to the linearized system, and should be avoided as much as possible in the analysis of the numerical model. Under the linear approximation, the amplitude of the perturbation can be canceled from all equations. Therefore, one obtains a dispersion relation that describes the intrinsic linear properties of the system. For the "numerical plasma" model in a PIC code, we will follow the guidelines established above and present a rigorous derivation of the full three dimensional, relativistic, numerical dispersion relation for the standard E.M. PIC model in this paper.

Our work is motivated by recent interest in the so-called "boosted frame" simulation technique $[9,10]$ where the simulation is conducted in a drifting cold plasma background and numerical instability has been observed to distort the results. Although the numerical Cherenkov instability $[11,12]$ is generally believed to be responsible for the unstable modes in the "boosted frame" simulation [13, 14], a more fundamental type of numerical instability - finite grid instability - may also be of interest for understanding this technique and other similar simulation models. In particular, the finite grid instability was first analyzed in Reference [1] for the explicit, momentum conserving and energy conserving Electrostatic (E.S.) PIC code.

More recently, a fully implicit energy and charge conserving E.S. PIC algorithm was developed and analyzed in Reference [15]. Rigorous energy conservation is enforced in this scheme, so the simulation is stable against the finite grid instability, while approximate momentum conservation is achieved from particle substepping. However, an analysis similar to that in Reference [1] for the E.M. PIC code is lacking, even for a fully explicit E.M. scheme. Thus, our work serves as a bridge between the numerical analysis of the E.S. and E.M. PIC models, as well as a framework for further analysis of the numerical instabilities in an E.M. PIC code.

Our reinvestigation of the finite grid instability shows that the above guidelines we seek to apply in this study may not have been fully appreciated in the past. As a result, we find that 1) The temporal aliasing effect should be absent in the PIC numerical dispersion relation. 2) The procedure in which the PIC model advances the particles' phase space distribution function can be exactly accounted for in the linear stability analysis. The effects of these new findings will be explored in detail in this paper.

This paper is organized as follows. In Section 2, we first review how aliasing arises in a hybrid system consisting of both discrete and continuous variables and its implication 
to the PIC model. Then, in Section 3, we describe the standard E.M. PIC model. In Section 4, we derive, in detail, the 3-D numerical dispersion relation for the standard E.M. PIC model, with a simple, direct current deposition scheme. After obtaining its accurate numerical dispersion relation, we investigate the finite grid instability in the E.M. PIC code for the 1-D case in Section 5. In this section, we focus on obtaining a detailed understanding of how such an instability occurs and its dependence on the choice of the numerical parameters. In Section 6, we show simulation verification of the finite grid instability and associated growth rates in 1-D for some numerical parameters of interest. In Section 7 we compare our analysis and results with previous treatments of similar, related problems, and discuss the finite grid instability in 1-D E.M. PIC with a charge conserving current deposition scheme. Finally, we summarize in Section 8.

\section{Aliasing}

A major difference between a continuous system and a discrete system is their Fourier space representations. If the system under consideration is inherently discrete (e.g., a crystal lattice), then all information about the system is contained in a single Brillouin zone (i.e., the uniquely defined primitive cell in Fourier space), with all other Brillouin zones being exact replicas. If, however, a discretization is imposed on a continuous system, with certain sampling frequency to obtain the discrete system, then every Brillouin zone must be considered to recover a complete description of the discretized system. This aliasing effect, which is used here in a general sense without considering the bandwidth of the continuous system relative to the Nyquist sampling frequency, is a major source of numerical instability in PIC and also a major complication in the instability analysis. In addition, it is worth noting that the aliasing effect only appears when one continuous system is sampled into a discrete one, not the other way around. Hence sampling is the only cause of aliasing in a numerical model.

In fact, both continuous and discrete variables are used in the PIC model, so all three scenarios described above are present. When particle quantities are deposited onto the spatial grid, an effective spatial sampling (but no temporal sampling) is done, since the particle distribution function and its derived information, such as the charge and the current, are actually known everywhere in space. However, a PIC code implementation does not need to generate the full information and can skip this step to evaluate charge or current only on the grid points. An extra complication exists due to the staggering of particles' positions and velocities in time, so that one can define various particle distribution functions depending on which time steps the position and velocity information are defined on. For example, a particular current deposition scheme may use one of many possible definitions of the particle distribution function. However, this is not the same as knowing the particle distribution function in a continuous time variable. When the field quantities on the grid are interpolated back to the particles' positions, a convolution process instead of a sampling is used. In Fourier space, this is equivalent to the multiplication of the Fourier contents of the fields and the convolution kernel, i.e., the Fourier transform of the particle form factor. For the temporal variable, one can readily recognize that the PIC model operates entirely in a discretized time, without the need to convert 
back and forth between continuous and discrete time variables in each computation loop.

It is also worth noting that, as time and space are two independent variables, aliasing in their respective Fourier conjugate variables are independent effects. An eigenmode in the PIC model consists of the particle eigen-distribution function, which is continuous in space, but discrete in time, and the eigenfields, which are discrete in both space and time. The aliasing effect in space will not lead to any aliasing effect in time because the only sampling in the PIC model occurs to the spatial variable during the charge and/or current deposition step. Therefore, when Fourier analyzing the eigenmodes in a PIC plasma, one should consider contributions from all spatial Brillouin zones. As no sampling is done to the temporal variable, the solutions in Fourier space to the PIC dispersion relation will be identical in each temporal Brillouin zone. Therefore, all physics of the system can be recovered by considering only the fundamental (zeroth) temporal Brillouin zone. It is sufficient to include only the contribution from this zone when constructing the Fourier space current in Section 4.

\section{The Electromagnetic PIC Model}

In this section, we briefly introduce the PIC model to be analyzed - the standard E.M. PIC model. In this E.M. PIC model, the Yee spatial grid is used for the fields.

We will use $\vec{r}_{A}$ to denote the position of a component of the vector field $\vec{A}$, and $\vec{r}^{\prime}$ to denote the continuous spatial variable. The field components are typically defined to be at either full or half integer grid points on the staggered Yee mesh. Whether a given component of a field is defined to be at a full or half integer grid point depends on the specific coordinate system adopted. Note that $\vec{r}_{A}$ will be different for each component of each field, but should be consistent with the coordinate system implementing the Yee mesh.

The equations of motion and Maxwell's equations are discretized with central differencing. Both the particles and the electromagnetic fields are advanced with the leap-frog scheme. In particular, particle position $\vec{r}$, velocity $\vec{v}$, and the fields $\vec{E}, \vec{B}$ are advanced in time according to ${ }^{1}$

$$
\begin{gathered}
\frac{d \vec{r}}{d t}=\vec{v} \longrightarrow \frac{\vec{r}_{n-1 / 2}-\vec{r}_{n-1}}{\Delta t / 2}=\vec{v}_{n-1 / 2} \text { and } \frac{\vec{r}_{n}-\vec{r}_{n-1 / 2}}{\Delta t / 2}=\vec{v}_{n-1 / 2}, \\
\frac{\partial \vec{E}}{\partial t}=\vec{\nabla} \times \vec{B}-4 \pi \vec{J} \longrightarrow \frac{\vec{E}_{n}-\vec{E}_{n-1}}{\Delta t}=\vec{\nabla} \times \vec{B}_{n-1 / 2}-4 \pi \vec{J}_{n-1 / 2}, \\
\frac{\partial \vec{B}}{\partial t}=-\vec{\nabla} \times \vec{E} \longrightarrow \frac{\vec{B}_{n+1 / 2}-\vec{B}_{n-1 / 2}}{\Delta t}=-\vec{\nabla} \times \vec{E}_{n}, \\
\frac{d(\gamma \vec{v})}{d t}=\frac{q}{m}(\vec{E}+\vec{v} \times \vec{B}) \longrightarrow \frac{(\gamma \vec{v})_{n+1 / 2}-(\gamma \vec{v})_{n-1 / 2}}{\Delta t}=\frac{q}{m}\left(\vec{E}_{n}+\vec{v}_{n} \times \vec{B}_{n}\right),
\end{gathered}
$$

where $\gamma=1 / \sqrt{1-v^{2}}, q, m$ are the particle Lorentz factor, charge, and mass, respectively. The luminal vacuum speed has been set to unity. The equations for the continuous system

\footnotetext{
${ }^{1}$ Note that splitting the time centered equation of motion into two equations, Eq. (1), is required to deposit the current at the centered time in the simple deposition scheme analyzed in Section 4.
} 
are shown on the left and their discrete analogs used in the PIC model are shown on the right. Here, $n$ indexes the time step, $t_{n}=n \Delta t$. With the Boris algorithm for particle pushing, Eq. (4) takes on the form of a pure rotation,

$$
\frac{(\gamma \vec{v})^{+}-(\gamma \vec{v})^{-}}{\Delta t}=\frac{q}{2 m \gamma_{n}}\left[(\gamma \vec{v})^{+}+(\gamma \vec{v})^{-}\right] \times \vec{B}_{n}
$$

and two half acceleration steps $(\gamma \vec{v})_{n \pm 1 / 2}=(\gamma \vec{v})^{ \pm} \pm q \vec{E}_{n} \Delta t / 2 m$. Equations (1)-(5) are implemented as shown in Figure 1.

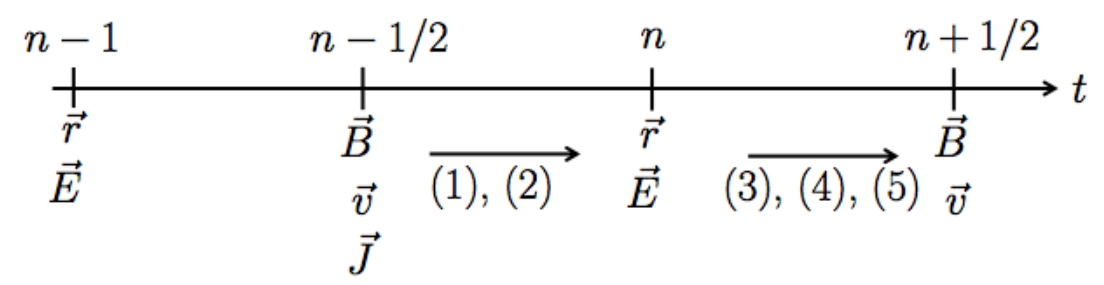

Figure 1: A schematic diagram of how the system is advanced in an E.M. PIC model. Suppose $\vec{r}_{n-1}, \vec{E}_{n-1}, \vec{v}_{n-1 / 2}$ and $\vec{B}_{n-1 / 2}$ are known. First, a half time step push is carried out using Eq. (1) to get $\vec{r}_{n-1 / 2}$, which is used with $\vec{v}_{n-1 / 2}$ to obtain the particle distribution function at time step $n-1 / 2$ and then $\vec{J}_{n-1 / 2}$. Equation (2) is used to get $\vec{E}_{n}$, and Eqs. (3), (4), and (5) are used to obtain $\vec{B}_{n+1 / 2}$ and $\vec{v}_{n+1 / 2}$. Another half push is then performed to get $\vec{r}_{n}$. The value of $\vec{B}_{n}$ in Eq. (4) is taken as an average from $\vec{B}_{n-1 / 2}$ and $\vec{B}_{n+1 / 2}$.

In Eq. (2), the current is calculated at the same grid points, $\vec{r}_{E}$, as where $\vec{E}$ is defined. This is exactly equivalent to spatial sampling, as the underlying particle distribution, from which the current is obtained, is defined on the continuous spatial variable. There are many current deposition schemes developed for the E.M. PIC code. Among those, the charge conserving schemes in [16] and [17] are widely used. Although the exact details of these schemes differ, one can attribute their differences to being the particle and velocity distribution and weighting function used in the deposition step. For example, some current deposition schemes use the concept of a "virtual particle" for the deposition step. The "virtual particles" are created through a transform on the actual particle distribution at one time step or actual distributions at several consecutive time steps (but not at an arbitrary continuous time so this is not a form of sampling in time). Similarly, other current deposition schemes define their own transforms.

In this paper, we ignore these differences in the transform and analyze the standard E.M. PIC code with a simple current deposition scheme where the particle distribution function is used directly to obtain the current at the same step (in Section 7, the analysis is extended to two charge conserving schemes). This is shown in Figure 1, where the particle distribution at $t_{n-1 / 2}$ is first obtained through a half position update and then the current is deposited. For this simple scheme, the current at its respective grid points, $\vec{J}\left(\vec{r}_{E}, t_{n-1 / 2}\right)$, is related to the current in the continuous space, $\vec{J}\left(\vec{r}^{\prime}, t_{n-1 / 2}\right)$, according to

$$
\vec{J}\left(\vec{r}_{E}, t_{n-1 / 2}\right)=\int \overleftrightarrow{S_{J}}\left(\vec{r}^{\prime}-\vec{r}_{E}\right) \cdot \vec{J}\left(\vec{r}^{\prime}, t_{n-1 / 2}\right) d \vec{r}^{\prime}
$$


Here, $\overleftrightarrow{S_{J}}\left(\vec{r}^{\prime}-\vec{r}_{E}\right)$ is the current interpolation tensor which is determined by the particle shape. For a more complicated current deposition scheme, the particle distribution transform will introduce an additional factor in the integral.

Note that, in Eq. (6), electric charge is, in general, not exactly conserved. Later, we will see that this observation is crucial to understanding the origin of a numerical instability predicted by the numerical dispersion relation.

\section{Derivation of the Numerical Dispersion Relation for the Electromagnetic PIC Code}

In this section, we will derive the numerical dispersion relation step by step.

\subsection{Numerical Wave Equation and Current Deposition in Fourier Space}

To obtain the numerical dispersion relation for the PIC model, we'll use the normalized dimensionless quantities for the electric field $\vec{E} \rightarrow e \vec{E} / m \omega_{p}$, magnetic field $\vec{B} \rightarrow e \vec{B} / m \omega_{p}$, temporal coordinate $t \rightarrow \omega_{p} t$, spatial coordinate $\vec{r} \rightarrow \omega_{p} \vec{r}$, momentum $\vec{p} \rightarrow \vec{p} / m$, temporal Fourier frequency $\omega \rightarrow \omega / \omega_{p}$, spatial Fourier frequency $\vec{k} \rightarrow \vec{k} / k_{p}$, electric charge $q \rightarrow q / e$, number density $n \rightarrow n / n_{p}$, and electric current $\vec{J} \rightarrow \vec{J} / e n_{p}$. Here, $m$ is the electron mass, $e$ is the elementary charge, $n_{p}$ is the electron plasma density, and $\omega_{p}=\sqrt{4 \pi e^{2} n_{p} / m}$ and $k_{p}$ are the electron plasma frequency and wavenumber.

We begin with the Ampère and Faraday laws in Fourier space,

$$
\begin{array}{r}
i \vec{k}_{B} \times \tilde{\vec{B}}=\tilde{\overrightarrow{\vec{J}}}-i \omega_{E} \tilde{\overrightarrow{\vec{E}}}, \\
\vec{k}_{E} \times \tilde{\vec{E}}=\omega_{B} \tilde{\vec{B}},
\end{array}
$$

where,

$$
\begin{aligned}
& \vec{E}\left(\vec{r}_{E}, t_{n-1}\right)=\tilde{\overrightarrow{\vec{E}}}(\vec{k}, \omega) e^{i\left(\vec{k} \cdot \vec{r}_{E}-\omega t_{n-1}\right)}, \\
& \vec{B}\left(\vec{r}_{B}, t_{n-1 / 2}\right)=\tilde{\vec{B}}(\vec{k}, \omega) e^{i\left(\vec{k} \cdot \vec{r}_{B}-\omega t_{n-1 / 2}\right)}, \\
& \vec{J}\left(\vec{r}_{E}, t_{n-1 / 2}\right)=\tilde{\vec{J}}(\vec{k}, \omega) e^{i\left(\vec{k} \cdot \vec{r}_{E}-\omega t_{n-1 / 2}\right)} .
\end{aligned}
$$

In Eqs. (7), $\tilde{\overrightarrow{\vec{E}}}, \tilde{\overrightarrow{\vec{B}}}$, and $\tilde{\vec{J}}$ are the spectral representations of the fields and current, and $\vec{k}_{E}, \vec{k}_{B}$, and $\omega_{E}, \omega_{B}$ are the spatial and temporal finite difference operators for the fields. These operators, of course, depend on the specific finite difference scheme employed by the field solver. We note that, in order to define $\vec{k}_{E}, \vec{k}_{B}$, and $\omega_{E}, \omega_{B}$ in their conventional forms, $\tilde{\vec{E}}, \tilde{\vec{B}}$, and $\tilde{\vec{J}}$ are not necessarily discrete Fourier transforms of the corresponding real space quantities. The true discrete Fourier transform of a function must be periodic across Brillouin zones, and this implies that a phase of zero must be present somewhere on the spatial and temporal grids where the function is defined. Because of this, there 
may be phase factors relating $\tilde{\vec{E}}, \tilde{\vec{B}}$, and $\tilde{\vec{J}}$ in different Brillouin zones. Combining Eqs. (7) yields the Fourier space numerical wave equation,

$$
\vec{k}_{B} \times \vec{k}_{E} \times \tilde{\vec{E}}+\omega_{B} \omega_{E} \tilde{\vec{E}}=-i \omega_{B} \tilde{\vec{J}}
$$

To evaluate Eq. (9), we need an expression for the current, $\tilde{\vec{J}}(\vec{k}, \omega)$. Let us assume a linearized kinetic model for a uniform, charge-neural and current-free plasma consisting of drifting cold electrons of charge $q_{e}$ and rest mass $m$, and co-moving neutralizing ions with infinite mass. For this model, we only consider the electron response. The distribution function for the electrons is

$$
f\left(\vec{r}^{\prime}, \vec{p}, t\right)=f_{0}(\vec{p})+f_{1}\left(\vec{r}^{\prime}, \vec{p}, t\right),
$$

with $\left|f_{1} / f_{0}\right|<<1$ and $\int f_{0}(\vec{p}) d \vec{p}=1$.

At time $t_{n-1 / 2}=(n-1 / 2) \Delta t$, the current in continuous real space is

$$
\vec{J}\left(\vec{r}^{\prime}, t_{n-1 / 2}\right)=q_{e} \int \frac{\vec{p}}{\gamma} f_{1}\left(\vec{r}^{\prime}, \vec{p}, t_{n-1 / 2}\right) d \vec{p}
$$

Combining Eqs. (6) and (11), it follows that

$$
\vec{J}\left(\vec{r}_{E}, t_{n-1 / 2}\right)=q_{e} \iint \overleftrightarrow{S_{J}}\left(\vec{r}^{\prime}-\vec{r}_{E}\right) \cdot \frac{\vec{p}}{\gamma} f_{1}\left(\vec{r}^{\prime}, \vec{p}, t_{n-1 / 2}\right) d \vec{p} d \vec{r}^{\prime}
$$

This is the expression for current deposition in the PIC model, where a spatial sampling is carried out implicitly. No temporal sampling is done as both sides of the equation are defined on the same discrete time. For the linear dispersion analysis presented here, the fully discretized current $\vec{J}\left(\vec{r}_{E}, t_{n-1 / 2}\right)$ can be written in the form of a monochromatic plane wave with discrete spatial and time dependence, i.e., as an eigenmode, as in Eq. (8).

Equation (12) also indicates that $f_{1}$ has the same dependence on $\omega$ as $\vec{J}\left(\vec{r}_{E}, t_{n-1 / 2}\right)$. On the other hand, one cannot assume that they have the same dependence on $\vec{k}$, due to aliasing resulting from spatial sampling. However, since $\vec{r}^{\prime}$ is continuous, we can write

$$
f_{1}\left(\vec{r}^{\prime}, \vec{p}, t_{n-1 / 2}\right)=e^{-i \omega t_{n-1 / 2}} \int \tilde{f}_{1}(\vec{k}, \vec{p}, \omega) e^{i \vec{k} \cdot \vec{r}^{\prime}} d \vec{k}
$$

and

$$
\overleftrightarrow{S_{J}}\left(\vec{r}^{\prime}\right)=\int \overleftrightarrow{S_{J}}(\vec{k}) e^{i \vec{k} \cdot \vec{r}^{\prime}} d \vec{k}
$$

From Eqs. (12) - (14), we obtain

$$
\tilde{\vec{J}}(\vec{k}, \omega)=q_{e} \int \tilde{S_{J}}\left(\vec{k}^{\prime}\right) \cdot\left[\int \frac{\vec{p}}{\gamma} \tilde{f}_{1}\left(\vec{k}^{\prime}, \vec{p}, \omega\right) d \vec{p}\right] \sum_{\vec{r}_{E}} e^{i\left(\vec{k}^{\prime}-\vec{k}\right) \cdot \vec{r}_{E}} d \vec{k}^{\prime}
$$

A straight forward application of Poisson's summation formula provides 


$$
\sum_{\vec{r}_{E}} e^{i\left(\vec{k}^{\prime}-\vec{k}\right) \cdot \vec{r}_{E}}=\sum_{\vec{q}} \delta\left(\vec{k}^{\prime}-\vec{k}+\vec{q}\right),
$$

with $\vec{q}=2 \pi\left(q_{x} / \Delta x, q_{y} / \Delta y, q_{z} / \Delta z\right)$ being a vector between grid points in the 3-D Fourier lattice. As a direct result

$$
\tilde{\vec{J}}(\vec{k}, \omega)=q_{e} \sum_{\vec{q}} \tilde{S_{J}}\left(\vec{k}_{q}\right) \cdot \int \frac{\vec{p}}{\gamma} \tilde{f}_{1}\left(\vec{k}_{q}, \vec{p}, \omega\right) d \vec{p}
$$

where $\vec{k}_{q}=\vec{k}-\vec{q}$, and the summation over $\vec{k}_{q}$ accounts for the spatial sampling. The wave equation, Eq. (9), now takes on the form

$$
\left.\vec{k}_{B} \times \vec{k}_{E} \times \tilde{\vec{E}}+\omega_{B} \omega_{E} \tilde{\overrightarrow{\vec{E}}}=-i q_{e} \omega_{B} \sum_{\vec{q}} \stackrel{\tilde{S_{J}}}{\left(\vec{k}_{q}\right.}\right) \cdot \int \frac{\vec{p}}{\gamma} \tilde{f}_{1}\left(\overrightarrow{k_{q}}, \vec{p}, \omega\right) d \vec{p}
$$

We obtain an expression for $\tilde{f}_{1}$ in the next section.

\subsection{Expression for the First Order Distribution Function in Fourier Space}

In a continuous system, the first order distribution function $f_{1}\left(\vec{r}^{\prime}, \vec{p}, t\right)$ in Eq. (10) satisfies the linearized Vlasov equation

$$
\frac{\partial f_{1}}{\partial t}+\frac{\vec{p}}{\gamma} \cdot \vec{\nabla} f_{1}+\vec{F} \cdot \vec{\nabla}_{p} f_{0}=0
$$

where $\vec{F}=q_{e}\left[\vec{E}\left(\vec{r}^{\prime}, t\right)+(\vec{p} / \gamma) \times \vec{B}\left(\vec{r}^{\prime}, t\right)\right]$ is the Lorentz force.

However, in the PIC model, Eq. (19) is solved in three steps, beginning from a particle distribution with position and velocity known at time step $n-1 / 2$ (after current deposition). The first step is a half time step position push, followed by a full time step momentum push, then another half time step position push. After these three steps, the particle distribution is advanced to the time step $n+1 / 2$. To account for this, we follow a procedure similar to what is implemented in Reference [18].

The first half position push is equivalent to solving

$$
\frac{\partial f_{1}}{\partial t}+\frac{\vec{p}}{\gamma} \cdot \vec{\nabla} f_{1}=0
$$

since momentum is fixed for this step, for a half time step. It is important to note that this is accomplished in PIC using the method of characteristics instead of Fourier transforming or using a finite difference approximation of Eq. (20). The set of particle trajectories at each time step form the characteristics of $f$ in phase space. If the first order distribution function used for the current deposition at time step $n-1 / 2$ is taken to be $f_{1}\left(\vec{r}^{\prime}, \vec{p}, t_{n-1 / 2}\right)=\tilde{f}_{1}(\vec{k}, \vec{p}, \omega) e^{i\left(\vec{k} \cdot \vec{r}^{\prime}-\omega t_{n-1 / 2}\right)}$, then after the half position push, the (partially) updated distribution function $f_{1}^{*}\left(\vec{r}^{\prime}, \vec{p}\right)=\tilde{f}_{1}^{*}(\vec{k}, \vec{p}, \omega) e^{i\left(\vec{k} \cdot \vec{r}^{\prime}-\omega t_{n-1 / 2}\right)}$ is

$$
f_{1}^{*}\left(\vec{r}^{\prime}, \vec{p}\right)=f_{1}\left(\vec{r}^{\prime}-\vec{v} \Delta t / 2, \vec{p}, t_{n-1 / 2}\right)
$$


where the time argument of $f_{1}^{*}$ has been omitted for clarity, since this distribution function is constructed using the particle position and velocity at different time steps. ${ }^{2}$ It follows that

$$
\tilde{f}_{1}^{*}(\vec{k}, \vec{p}, \omega)=\tilde{f}_{1}(\vec{k}, \vec{p}, \omega) e^{-i \vec{k} \cdot \vec{v} \Delta t / 2} .
$$

Next we look at the full momentum push, which is equivalent to solving

$$
\frac{\partial f_{1}}{\partial t}+\vec{F} \cdot \vec{\nabla}_{p} f_{0}(\vec{p})=0
$$

since position is fixed, for a full time step. This is accomplished in PIC using the Boris pusher and can be viewed as a transformation of the distribution function between old momentum $\vec{p}^{\prime}$ and the updated one $\vec{p}$. It can be shown that the determinant of the Jacobian is unity for the transform under linearization of the Lorentz force, i.e., $\vec{F}=$ $q_{e}\left[\vec{E}\left(\vec{r}^{\prime}, t\right)+\vec{v}_{0} \times \vec{B}\left(\vec{r}^{\prime}, t\right)\right]$, where $\vec{v}_{0}$ is the unperturbed velocity. Now, the updated distribution $f_{1}^{* *}\left(\vec{r}^{\prime}, \vec{p}\right)$ can be written as

$$
f_{1}^{* *}\left(\vec{r}^{\prime}, \vec{p}\right)=\int\left[f_{0}\left(\vec{p}^{\prime}\right)+f_{1}^{*}\left(\vec{r}^{\prime}, \vec{p}^{\prime}\right)\right] \delta\left(\vec{p}-\vec{p}^{\prime}-\Delta \vec{p}\right) d \vec{p}^{\prime}-f_{0}(\vec{p}),
$$

with $\Delta \vec{p}=\vec{F} \Delta t^{3}$

Taylor expanding the $\delta$-function about $\vec{p}=\vec{p}^{\prime}$, linearizing, then integrating by parts gives

$$
f_{1}^{* *}\left(\vec{r}^{\prime}, \vec{p}\right) \approx-\int \Delta \vec{p} \cdot \vec{\nabla}_{p^{\prime}} f_{0}\left(\vec{p}^{\prime}\right) \delta\left(\vec{p}-\vec{p}^{\prime}\right) d \vec{p}^{\prime}+\int f_{1}^{*}\left(\vec{r}^{\prime}, \vec{p}^{\prime}\right) \delta\left(\vec{p}-\vec{p}^{\prime}\right) d \vec{p}^{\prime},
$$

or,

$$
f_{1}^{* *}\left(\vec{r}^{\prime}, \vec{p}\right) \approx-\vec{\nabla}_{p} f_{0}(\vec{p}) \cdot \Delta \vec{p}+f_{1}^{*}\left(\vec{r}^{\prime}, \vec{p}\right) .
$$

We see that $\vec{F}$ is equal to the sum of two terms - one involving the perturbation $\vec{E}$, and one involving the perturbation $\vec{B}$. Both terms are expressed as the discrete eigenmodes in Eq. (8), so we must have $\vec{F}\left(\vec{r}^{\prime}, \vec{p}, t_{n}\right)=\tilde{\vec{F}}(\vec{k}, \vec{p}, \omega) e^{i\left(\vec{k} \cdot \vec{r}^{\prime}-\omega t_{n-1 / 2}\right)}$. (Note that we use the same time harmonic form $e^{-i \omega t_{n-1 / 2}}$ as in $\vec{J}\left(\vec{r}_{E}, t_{n-1 / 2}\right)$ for $\vec{F}\left(\vec{r}^{\prime}, \vec{p}, t_{n}\right)$, and leave the discussion of the spatial and temporal phase factors relating $\vec{E}, \vec{B}$, and $\vec{F}$ to the next section.) Therefore,

$$
\tilde{f}_{1}^{* *}(\vec{k}, \vec{p}, \omega)=-\Delta t \tilde{\vec{F}}(\vec{k}, \vec{p}, \omega) \cdot \vec{\nabla}_{p} f_{0}(\vec{p})+\tilde{f}_{1}(\vec{k}, \vec{p}, \omega) e^{-i \vec{k} \cdot \vec{v} \Delta t / 2} .
$$

For the second half position push, we solve for the updated distribution function like we did for the first half push. The (fully) updated distribution $f_{1}^{* * *}$ becomes

$$
f_{1}^{* * *}\left(\vec{r}^{\prime}, \vec{p}\right)=f_{1}^{* *}\left(\vec{r}^{\prime}-\vec{v} \Delta t / 2, \vec{p}\right) .
$$

\footnotetext{
${ }^{2}$ The function $f_{1}^{*}$ is not a physical distribution, as it is not actually defined on a single time step. It is just an intermediary function that arises when calculating the physical distribution $f_{1}$, which is defined at a single time step.

${ }^{3}$ Note that $\vec{p}$ is the momentum axis coordinate and is continuous. It is not the individual particles' momenta and is not a function of the discrete time coordinate.
} 
Combining Eqs. (22), (27) and (28), we obtain

$$
\tilde{f}_{1}^{* * *}(\vec{k}, \vec{p}, \omega)=-\Delta t \tilde{\vec{F}}(\vec{k}, \vec{p}, \omega) \cdot \vec{\nabla}_{p} f_{0} e^{-i \vec{k} \cdot \vec{v} \Delta t / 2}+\tilde{f}_{1}(\vec{k}, \vec{p}, \omega) e^{-i \vec{k} \cdot \vec{v} \Delta t}
$$

Since one full time step has elapsed after these three steps, $\tilde{f}_{1}^{* * *}(\vec{k}, \vec{p}, \omega)=\tilde{f}_{1}(\vec{k}, \vec{p}, \omega) e^{-i \omega \Delta t}$. Therefore,

$$
\tilde{f}_{1}(\vec{k}, \vec{p}, \omega)=\frac{-i e^{i \omega \Delta t / 2}}{2 / \Delta t} \csc \{(\omega-\vec{k} \cdot \vec{v}) \Delta t / 2\} \tilde{\vec{F}}(\vec{k}, \vec{p}, \omega) \cdot \vec{\nabla}_{p} f_{0},
$$

where we have used that $e^{-i \vec{k} \cdot \vec{v} \Delta t / 2} /\left(e^{-i \vec{k} \cdot \vec{v} \Delta t}-e^{-i \omega \Delta t}\right)=-i e^{i \omega \Delta t / 2} \csc \{(\omega-\vec{k} \cdot \vec{v}) \Delta t / 2\} / 2$.

Equation (30) includes the effect of a finite time step on $\tilde{f}_{1}$. Clearly, $\lim _{\Delta t \rightarrow 0} \tilde{f}_{1}=$ $-i \tilde{\vec{F}} \cdot \vec{\nabla}_{p} f_{0} /(\omega-\vec{k} \cdot \vec{v})$, which recovers the expression for a system with a continuous time variable. Now we are in need of an expression for $\tilde{\vec{F}}$, the linearized Lorentz force. We find this in the following section by paying careful attention to the spatial and temporal phase factors.

\subsection{Expression for the Lorentz Force in Fourier Space}

Before we proceed, it is important to ensure that all the terms in a given spatial component of an equation are evaluated using the same reference time step and the same reference spatial grid. Indeed, with the use of a Yee grid for the fields, Eq. (7) implies that each spatial component of these equations is defined at the same grid points (i.e., the reference phase for each component on its respective grid has been factored out) as the field not involved in the spatial derivative (e.g., the $x$ component of Faraday's law is defined at the same grid points as $B_{x}$, and the $x$ component of Ampère's law is defined at the same grid points as $E_{x}$, etc.).

For the remainder of this section, we choose the reference grid to be different for each component of the wave equation, Eq. (9). The integer grid points in a component $\alpha$ of Eq. (9) will be located at the points where the component $\alpha$ of $\vec{E}$ is defined. With this choice of coordinates, one can show that the $\beta$ component of the fields, in the fundamental Brillouin zone, appearing in the $\alpha$ component of Eq. (9), is related to the same component of fields outside of the fundamental zone by $\tilde{\vec{E}}\left(\overrightarrow{k_{q}}, \omega\right)=\overleftrightarrow{O_{E}} \cdot \tilde{\vec{E}}(\vec{k}, \omega)$ and $\tilde{\vec{B}}\left(\vec{k}_{q}, \omega\right)=\overleftrightarrow{O_{B}} \cdot \tilde{\vec{B}}(\vec{k}, \omega)$, where

$$
O_{E, \alpha}^{\beta \beta}=\left\{\begin{array}{ll}
1, & \text { if } \alpha=\beta \\
(-1)^{q_{\alpha}+q_{\beta},}, & \text { if } \alpha \neq \beta
\end{array} \text { and } O_{B, \alpha}^{\beta \beta}=\left\{\begin{array}{ll}
(-1)^{q_{\alpha}+q_{\beta}+q_{\zeta},} & \text { if } \alpha=\beta \\
(-1)^{q_{\zeta},} & \text { if } \alpha \neq \beta
\end{array},\right.\right.
$$

where $\zeta$ denotes the direction that is not $\alpha$ or $\beta$, and off diagonal terms are zero. We emphasize that $\vec{r}_{E}$ is different for each component of $\vec{J}$, since the components of $\vec{E}$ are defined at different points in space. The variation in where quantities are defined causes the elements in Eq. (31) to be different than unity. For example, $O_{E, x}^{y y}=(-1)^{q_{x}+q_{y}}$ in $\overleftrightarrow{O_{E}}$ arises because the $y$ component of $\vec{E}$ is offset from the $x$ component by $\Delta x / 2$ in the 
$x$ direction, and $\Delta y / 2$ in the $y$ direction in real space. In Fourier space, this implies that $\tilde{E}_{y}=\tilde{E}_{y}^{\prime} e^{i\left(k_{x} \Delta x / 2+k_{y} \Delta y / 2\right)}$, where $\tilde{E}_{y}^{\prime}$ is the discrete Fourier transform of $E_{y}$, and is identical in each Brillouin zone, while $\tilde{E}_{y}$ is not.

It is conventional to define the transformed finite difference operators $\omega_{B}=\omega \operatorname{sinc}(\omega \Delta t / 2)$ and $\vec{k}_{E}=\left[k_{x} \operatorname{sinc}\left(k_{x} \Delta x / 2\right), k_{y} \operatorname{sinc}\left(k_{y} \Delta y / 2\right), k_{z} \operatorname{sinc}\left(k_{z} \Delta z / 2\right)\right]$ in Faraday's law, because the standard PIC model uses a centered scheme when calculating derivatives. ${ }^{4}$ Similarly, for Ampère's law in Eq. (7), we choose $\omega_{E}=\omega_{B}$ and $\vec{k}_{B}=\vec{k}_{E}$. We will explicitly account for any implicit phase factors that may be present due to the staggered temporal and spatial grids, the choice of the reference phase, and the form of the finite difference operators.

In Ampère's law from Eq. (7), $\tilde{\vec{J}}$ is calculated using the components of $\vec{J}$ evaluated at the time step of $\vec{B}$ and the spatial grids of the components of $\vec{E}$. The expression for $\vec{J}$ is given by Eq. (12), and is proportional to $f_{1}$ at the same time step. Furthermore, $f_{1}$ is proportional to the Lorentz force, which involves $\vec{E}$ and $\vec{B}$, for which the same reference phase for the time step and grid should be used. However, according to Figure 1, the leap-frog advance requires the Lorentz force to be evaluated a half time step ahead of $f_{1}$ and $\vec{B}$. The field $\vec{E}$ is defined on this time step so it does not require time averaging, but a temporal phase factor is needed to account for the half time step difference relative to $f_{1}$. The field $\vec{B}$ is not defined on this time step and thus requires time averaging. In order to account for the spatial phases factored out from the components of Eq. (7) and thus the Lorentz force, one also needs to consider the spatial phase difference between the staggered spatial grids relative to that of the corresponding component of $\vec{E}$. The expression for the Lorentz force, which is extrapolated to a particle's position $\vec{r}$ and includes all the phase factors mentioned above, is given by

$$
\begin{aligned}
\vec{F}\left(\vec{r}, \vec{p}, t_{n}\right) & =\tilde{\vec{F}}(\vec{k}, \vec{p}, \omega) e^{i\left(\vec{k} \cdot \vec{r}-\omega t_{n-1 / 2}\right)} \\
& =q_{e} \sum_{\vec{r}_{E}} e^{i\left(\vec{k} \cdot \vec{r}_{E}-\omega t_{n-1 / 2}\right)}\left\{\tau_{E} \overleftrightarrow{S_{E}}\left(\vec{r}-\vec{r}_{E}\right) \cdot \overleftrightarrow{\phi}_{E} \cdot \tilde{\vec{E}}+\tau_{B} \frac{\vec{p}}{\gamma} \times\left[\overleftrightarrow{S_{B}}\left(\vec{r}-\vec{r}_{B}\right) \cdot \overleftrightarrow{\phi}_{B} \cdot \tilde{\vec{B}}\right]\right\}
\end{aligned}
$$

where the temporal phase factors are $\tau_{E}=e^{-i \omega \Delta t / 2}$ and $\tau_{B}=\left(e^{-i \omega \Delta t}+1\right) / 2$, which results from time averaging. These are the temporal phase factors which allow us to factor $e^{-i \omega t_{n-1 / 2}}$ (i.e., the temporal phase of $\vec{B}$ ) from both sides of Ampère's in Eq. (7).

The presence of the spatial phase tensors $\overleftrightarrow{\phi}_{E}$ and $\overleftrightarrow{\phi}_{B}$ is short lived. In evaluating the sum over $\vec{r}_{E}$ in Eq. (32) to obtain an expression for $\tilde{\vec{F}}$ in terms of the transformed interpolation functions, $\tilde{\overrightarrow{S_{E}}}(\vec{k})$ and $\tilde{\overrightarrow{S_{B}}}(\vec{k})$, these factors vanish.

From Eqs. (7), (31), and (32)

$$
\left.\tilde{\vec{F}}\left(\overrightarrow{k_{q}}, \vec{p}, \omega\right)=q_{e}\left\{\tau_{E} \stackrel{\widetilde{S_{E}}}{\left(\overrightarrow{k_{q}}\right.}\right) \cdot \overleftrightarrow{O_{E}} \cdot \tilde{\vec{E}}(\vec{k}, \omega)+\frac{\tau_{B}}{\gamma \omega_{B}} \vec{p} \times \overleftrightarrow{\vec{S}_{B}}\left(\overrightarrow{k_{q}}\right) \cdot\left[\overleftrightarrow{O_{B}} \cdot \vec{k}_{E} \times \tilde{\vec{E}}(\vec{k}, \omega)\right]\right\}
$$

\footnotetext{
${ }^{4}$ For a non-standard PIC model, such as in Reference [19], the forms of these operators are different, and thus affect the numerical dispersion and instability. The numerical dispersion analysis of these algorithms will be presented in a future publication.
} 
We finally have an explicit expression for the current

$$
\tilde{\vec{J}}(\vec{k}, \omega)=q_{e} \frac{-i e^{i \omega \Delta t / 2}}{2 / \Delta t} \sum_{\vec{q}} \stackrel{\tilde{S}}{S}_{J}\left(\vec{k}_{q}\right) \cdot \int \frac{\vec{p}}{\gamma} \csc \left\{\left(\omega-\overrightarrow{k_{q}} \cdot \vec{v}\right) \Delta t / 2\right\} \tilde{\vec{F}}\left(\overrightarrow{k_{q}}, \vec{p}, \omega\right) \cdot \vec{\nabla}_{p} f_{0} d \vec{p}
$$

Equation (34) is similar to Eq. (10) in Reference [13], but we have accounted for the exact procedure for the distribution update in PIC and omitted a temporal aliasing effect. Note that these derivations are not equivalent, as they will produce different results when applied to other, similar, numerical models, such as a finite difference Vlasov model.

Substituting Eq. (34) into the Fourier space wave equation Eq. (9), we obtain an equation of the form

$$
\overleftrightarrow{\epsilon} \cdot \tilde{\vec{E}}(\vec{k}, \omega)=0
$$

This has nontrivial solutions for $\tilde{\vec{E}}(\vec{k}, \omega)$ only if

$$
\operatorname{Det}|\overleftrightarrow{\epsilon}|=0
$$

Equation (36) is the numerical dispersion relation relating $\vec{k}$ and $\omega$.

\section{$5 \quad$ Numerical Instability of the Discrete Eigenmodes for a Relativistically Drifting Cold Plasma}

Let us consider the case where the unperturbed distribution function is that for a cold relativistically drifting plasma. For the simplest case, the drift can be taken to be in the $\hat{x}$ direction with each electron having momentum of magnitude $p_{0}$, i.e.,

$$
f_{0}(\vec{p})=\delta\left(p_{x}-p_{0}\right) \delta\left(p_{y}\right) \delta\left(p_{z}\right) .
$$

In the case of a 1-D Yee scheme, the dispersion relation Eq. (36) becomes

$$
\left|\begin{array}{ccc}
\omega_{B} \epsilon_{a} & 0 & 0 \\
0 & \epsilon_{b} & 0 \\
0 & 0 & \epsilon_{b}
\end{array}\right|=0
$$

where

$$
\begin{aligned}
& \epsilon_{a}=\frac{\sin (\omega \Delta t / 2)}{\Delta t / 2}-\frac{4 \Delta t}{\gamma^{3}} \sin ^{4}(k \Delta x / 2) \times \\
& \sum_{q=-\infty}^{\infty} \frac{2 \sin \left\{\left(\omega-k_{q} v_{0}\right) \Delta t / 2\right\}+k_{q} v_{0} \Delta t \cos \left\{\left(\omega-k_{q} v_{0}\right) \Delta t / 2\right\}}{k_{q}^{4} \Delta x^{4}} \csc ^{2}\left\{\left(\omega-k_{q} v_{0}\right) \Delta t / 2\right\}
\end{aligned}
$$


and

$$
\begin{gathered}
\epsilon_{b}=\frac{\sin ^{2}(\omega \Delta t / 2)}{(\Delta t / 2)^{2}}-\frac{\sin ^{2}(k \Delta x / 2)}{(\Delta x / 2)^{2}}+\frac{16}{\gamma \Delta x} \sin ^{4}(k \Delta x / 2) \times \\
\sum_{q=-\infty}^{\infty} \csc \left\{\left(\omega-k_{q} v_{0}\right) \Delta t / 2\right\} \times \\
\frac{(-1)^{q} v_{0} \Delta t \cos (\omega \Delta t / 2) \sin (k \Delta x / 2)-\Delta x \sin (\omega \Delta t / 2)}{k_{q}^{4} \Delta x^{4}},
\end{gathered}
$$

and

$$
\omega_{B}=\frac{\sin (\omega \Delta t / 2)}{\Delta t / 2}
$$

with $k_{q}=k-2 \pi q / \Delta x, v_{0}=p_{0} / \gamma_{0}$, and $\gamma_{0}=1 / \sqrt{1-v_{0}^{2}}$. Note that the subscripts denoting directions have been omitted since only one direction is being considered. Equation (38) is clearly satisfied when any of the following conditions are met $\left(\omega_{B}=0\right.$ only gives $\omega=0$ in the fundamental temporal Brillouin zone)

$$
\begin{aligned}
\epsilon_{a} & =0, \\
\epsilon_{b} & =0 .
\end{aligned}
$$

In the limit we take $\Delta x \rightarrow 0, \Delta t \rightarrow 0$, and include only $q=0$ in the sum, Eqs. (42) and (43) become the dispersion equations for the electrostatic-like (E.S.-like) and electromagnetic-like (E.M.-like) modes of a drifting cold plasma. ${ }^{5}$ They are, respectively,

$$
\begin{aligned}
\omega^{2}-k^{2} & =1 / \gamma, \\
\left(\omega-k v_{0}\right)^{2} & =1 / \gamma^{3} .
\end{aligned}
$$

With a finite grid size and time step, Eqs. (42) and (43) are transcendental in $\omega$ and $k$ and must be solved numerically. For this paper, we will focus on the E.S.-like mode dispersion relation, Eq. (42). Before we delve into solving the full numerical dispersion relation, we consider the simpler case with $\Delta t \rightarrow 0$ and only a finite grid size $\Delta x>0$.

\subsection{Instability from the Electrostatic-like Mode with an In- finitesimal Time Step}

It is well known that PIC codes exhibit a type of numerical instability due to the use of a finite size grid. The analysis of this instability can be made using the drifting cold plasma model in the limit of an infinitely small time step size [1]. Taking this limit $\lim _{\Delta t \rightarrow 0} \epsilon_{a}=0$, we find that the numerical dispersion relation for the E.S.-like mode becomes

$$
\left(\omega_{t}+k v_{0}\right)\left[1-\frac{16 \Delta x^{2}}{\gamma^{3}} \sin ^{4}(k \Delta x / 2) \sum_{q=-\infty}^{\infty} \frac{1}{(k \Delta x-2 \pi q)^{4}\left(\omega_{t} \Delta x+2 \pi q v_{0}\right)^{2}}\right]=0
$$

\footnotetext{
${ }^{5}$ We add the "-like" suffix, because for $\gamma>1$ the E.S.-like eigenvector is not parallel to the wave vector. Similarly, the second mode is E.M.-like, as its eigenvector is not perpendicular to its wave vector for $\gamma>1[20]$.
} 
where $\omega_{t}=\omega-k v_{0}$. The first factor corresponds to a second standalone $\omega=0$ mode and is not of particular interest for the $\Delta t \rightarrow 0$ case, but it turns out to be important for the $\Delta t>0$ case, which will be discussed in Section 5.2. The infinite sum is easily evaluated with Cauchy's residue theorem.

We now set $\omega_{t}=\omega_{r}+i \omega_{i}$, and numerically search for solutions in the complex plane, given a set of numerical parameters $k, \Delta x$, and $v_{0}$. Figure 2 shows the solutions for $\omega_{r}$ and $\omega_{i}$ as functions of $k \Delta x$ with $v_{0}=0.1$. In general, the solutions for $\omega_{r}$ consist of a finite grid size plasma mode and alias modes corresponding to the poles from the sum in the second factor in Eq. (44). Therefore, they can be labeled by the index $q$ of the pole (the $q=0$ mode is the finite grid size plasma E.S.-like mode, while $q \neq 0$ modes are alias modes). The vertical location of a pole is determined by $\omega_{r}=-2 \pi q v_{0} / \Delta x$, and these poles form a set of parallel lines in the $\omega_{r}-k \Delta x$ plane. Each alias remains in the vicinity of the corresponding pole location. Therefore, the aliases are all roughly parallel to each other and also to the $k \Delta x$-axis, as seen in Figure 2 (a), (c), and (e). They may not cross each other, however, each mode also has one upper and one lower branch, which extend above and below its vertical location. (We note that a similar observation applies to the E.S. PIC dispersion relation Eq. (7) derived in chapter 8-11 of Reference [1] for $\Delta t \rightarrow 0$, but solving this for typical parameter values shows that each alias mode from this dispersion relation is itself unstable.) In Figure 2, it can be seen in that although each mode is stable $\left(\omega_{i}=0\right)$ by itself for small $\Delta x, \omega_{i} \neq 0$ for sufficiently large $\Delta x$. This is due to the overlapping of branches from adjacent modes as the vertical locations of the alias modes are lowered. Physically, such overlapping can result in unstable modes, for which $\omega_{i}>0$ (or $\omega_{i}<0$ ) corresponds to a growing (or damped) mode. However, one should distinguish the instability caused by overlapping of the branches of parallel aliases modes from that caused by the intersection of non-parallel modes, such as in the classic cold plasma two stream instability, which has no threshold.

Figure 2 (b), (d), and (f) indicates that the instability first develops and its growth rate remains largest in magnitude near $k \Delta x= \pm \pi$ as $\Delta x$ increases. This can be understood from Figure 2 (a), (c), and (e), of $\omega_{r}$, which shows that the branches of the $q=0$ mode, i.e., the finite grid size E.S.-like mode, $\omega_{r}=\omega_{\mathrm{ES}}^{ \pm} \approx \pm \operatorname{sinc}^{2}(k \Delta x / 2) / \gamma^{3 / 2}$ and the branches of the $q=\mp 1$ aliases first intersect at $k \Delta x= \pm \pi$ for $\Delta x \gtrsim 0.5625$. A second instability near $k \Delta x \approx 0$ is observed in Figure 2 (f) when comparing Figure 2 (d) and (f), although the associated instability is not as significant.

As discussed above, Figure 2 also indicates that there exists a threshold grid spacing, above which an unstable mode will be present. To obtain this threshold grid spacing and growth rate of the unstable modes near the threshold, we can analytically solve for $\omega_{t}$ near the intersection of the nearest alias mode with $\omega_{\mathrm{ES}}^{ \pm}$. Since the solution $\omega_{t}$ is anti-symmetric with respect to $k \Delta x$, without loss of generality, we can consider the intersection of $\omega_{\mathrm{ES}}^{-}$with the $q=1$ alias where the $q=0,1$ terms will dominate the sum in Eq. (44). Therefore, to a good approximation, $\omega_{t}$ satisfies

$$
1-\frac{16 \Delta x^{2}}{\gamma^{3}} \sin ^{4} \frac{k \Delta x}{2}\left[\frac{1}{\omega_{t}^{2} k^{4} \Delta x^{6}}+\frac{1}{(2 \pi-k \Delta x)^{4}\left(2 \pi v_{0}+\omega_{t} \Delta x\right)^{2}}\right] \approx 0\left(\omega_{r}<0\right) .
$$

It is possible to solve Eq. (45) analytically for $\omega_{t}$. Unfortunately, the solutions 

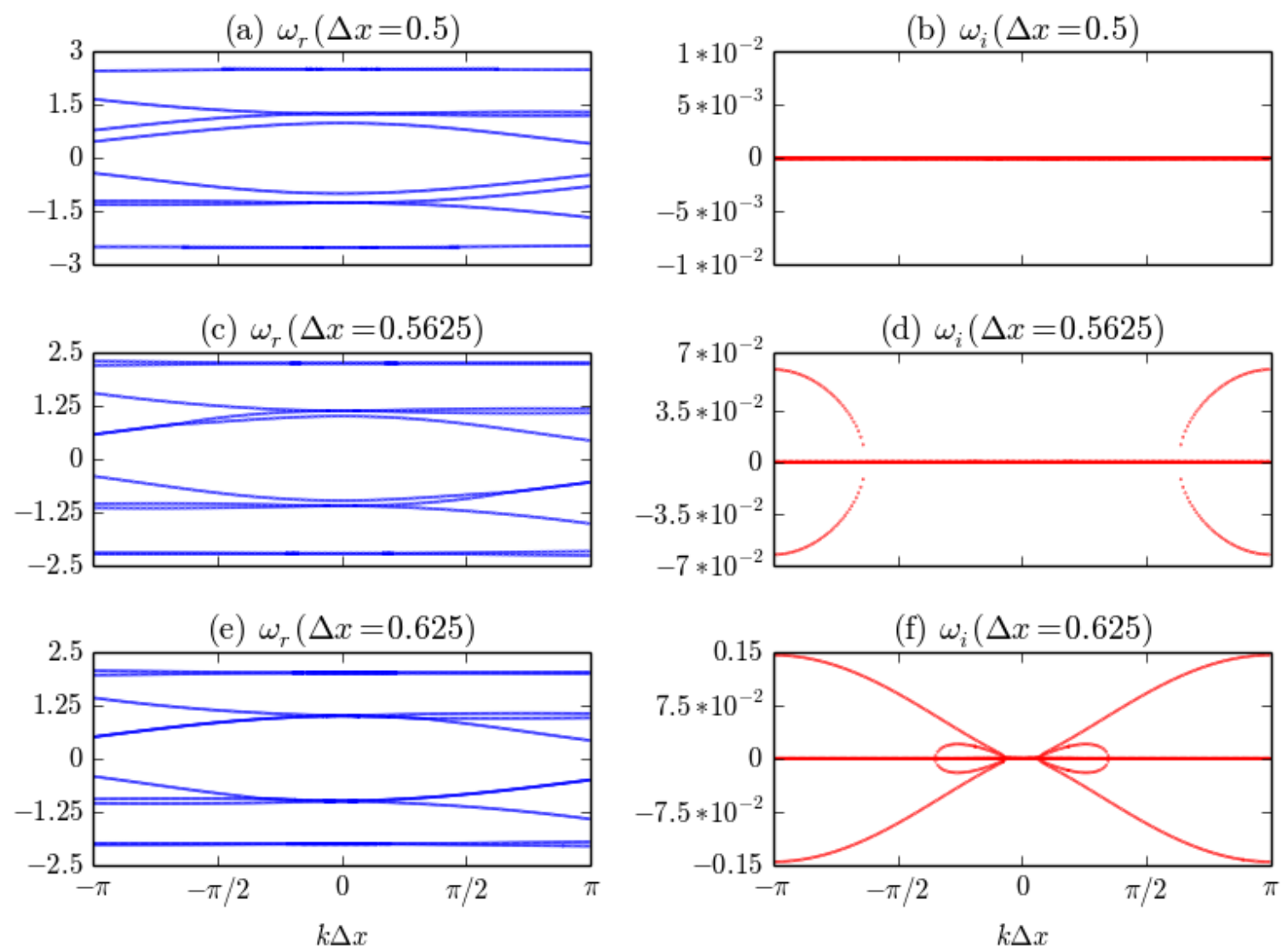

Figure 2: Numerical solutions to the dispersion relation Eq. (44) in the fundamental spatial Brillouin zone $k \Delta x \in[-\pi, \pi]$, for $v_{0}=0.1$ and three different values of $\Delta x$. $\Delta x=0.5,0.5625,0.625$ for the top, middle, and bottom rows, respectively. In (a), (c) and (e), the modes from top to bottom are the $q=-2,-1,0^{+}, 0^{-}, 1,2$ modes, where the $q=0^{ \pm}$modes are $\omega_{\mathrm{ES}}^{ \pm}$.

to Eq. (45) are very complicated. They are of little practical use and are not given here. However, a comparison of the analytic solutions to the approximated dispersion relation Eq. (45) against the numerical solutions of the exact dispersion relation Eq. (44), presented in plots (c) and (d) of Figure 2, is shown in Figure 3 to demonstrate the accuracy of the analytic solutions. There are three solutions to Eq. (45). There is one stable mode, shown in Figure 3 (a) and (b), and two unstable modes, one growing in Figure 3 (c) and (d), and one damped in Figure 3 (e) and (f).

Figure 3 (d) and (f) shows that the unstable modes are the upper branch of the $q=1$ alias, and the $\omega_{\mathrm{ES}}^{-}$branch of the finite grid size plasma mode. The lower branch of the $q=1$ alias in Figure 3 (a) is predicted to be stable. Since both $\omega_{r}$ and $\omega_{i}$ plots are anti-symmetric with respect to $k \Delta x$ and the upper branch of the $q=1$ alias in Figure 3 (c) is associated with the upper right lobe in Figure 3 (d), the lower branch of the $q=-1$ alias will then be associated with the lower left lobe of $\omega_{i}$ and is a damped mode. Similarly, the imaginary part of the $\omega_{\mathrm{ES}}^{+}$branch corresponds to the upper left lobe in 
the $\omega_{i}$ plots in Figure 3 and is a growing mode, while the upper branch of the $q=-1$ alias is a stable mode. Solving the $q=0,-1$ analog of Eq. (45) indeed verifies these observations.
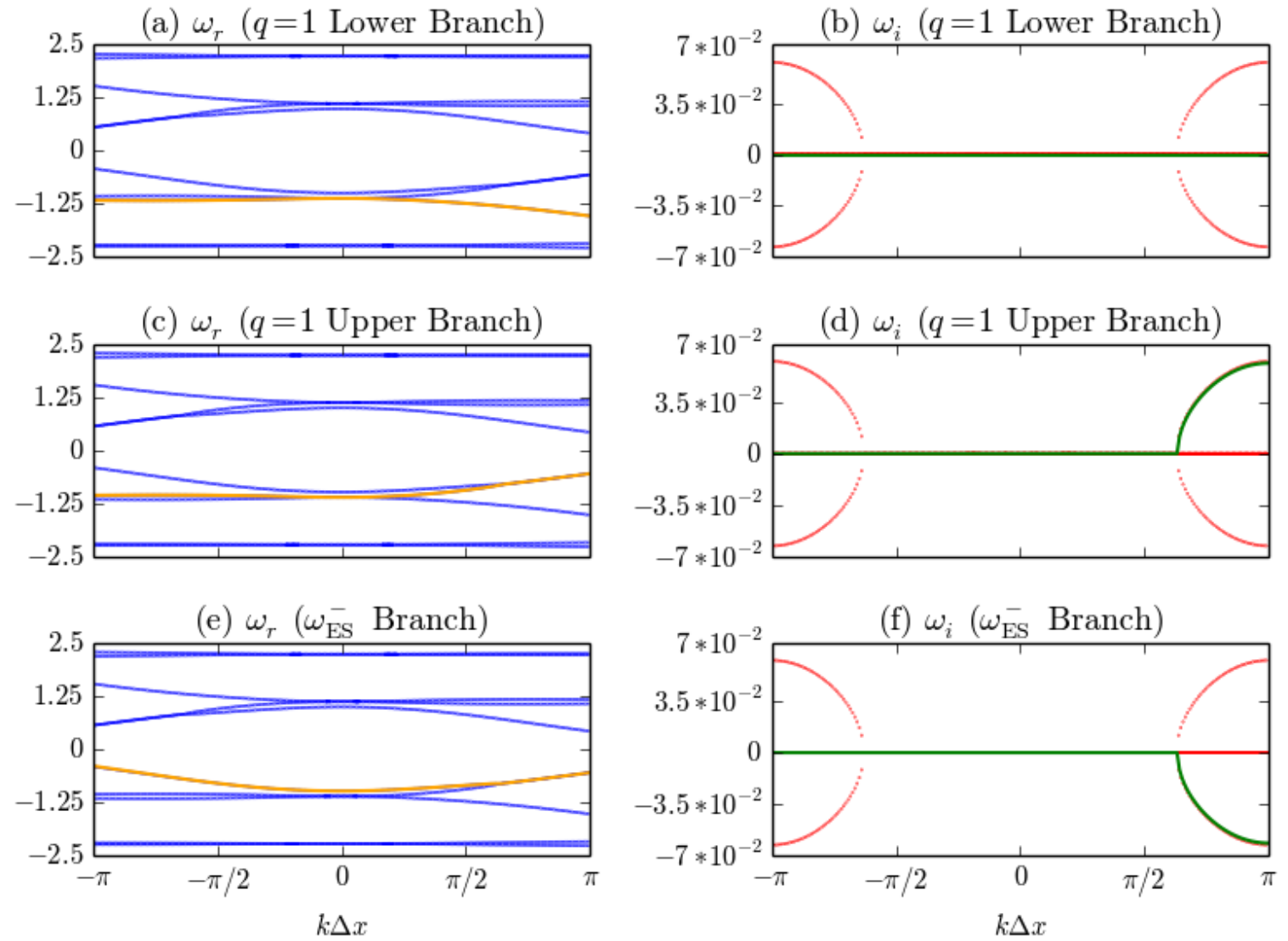

Figure 3: Analytic solutions to Eq. (45) (orange, green) and numerical solutions to Eq. (44) (blue, red) for $v_{0}=0.1$ and $\Delta x=0.5625$. Plots in the left column show real parts of solutions, and plots in the right column show imaginary parts. All numerical roots are present in each set of plots, while each of the three analytic roots from Eq. (45) are shown in separate rows, respectively. The plots are labeled according to the analytic root shown. The stable branch of the $q=1$ alias is shown in (a) and (b), and the unstable branch is shown in (c) and (d). The unstable branch of the finite grid E.S.-like mode is shown in (e) and (f).

As we are most interested in when an instability occurs and its peak growth rate, solving Eq. (45) evaluated at $k \Delta x=\pi$ (and considering all solutions) gives

$$
\omega_{t}(k \Delta x=\pi)=\left\{\begin{array}{l}
-\frac{\pi v_{0}}{\Delta x} \pm \sqrt{16 \pi^{-4} \gamma^{-3}+\left(\frac{\pi v_{0}}{\Delta x}\right)^{2}+8 \pi^{-2} \gamma^{-3} \sqrt{4 \pi^{-4}+\left(\frac{\pi v_{0}}{\Delta x}\right)^{2} \gamma^{3}}} \\
-\frac{\pi v_{0}}{\Delta x} \pm \sqrt{16 \pi^{-4} \gamma^{-3}+\left(\frac{\pi v_{0}}{\Delta x}\right)^{2}-8 \pi^{-2} \gamma^{-3} \sqrt{4 \pi^{-4}+\left(\frac{\pi v_{0}}{\Delta x}\right)^{2} \gamma^{3}}}
\end{array} .\right.
$$

We conclude that the maximum $\Delta x$ that will result in completely real solutions to 
Eq. (44) (i.e., for the term inside the square root of the second expression in Eq. (46) to be non-negative) is

$$
\Delta x_{\mathrm{th}}=\frac{\pi^{3} \gamma^{3 / 2} v_{0}}{4 \sqrt{2}}
$$

For larger $\Delta x$, the most unstable mode has an imaginary part with magnitude ${ }^{6}$

$$
\left|\omega_{i}(k \Delta x= \pm \pi)\right|=\sqrt{\left|16 \pi^{-4} \gamma^{-3}+\left(\frac{\pi v_{0}}{\Delta x}\right)^{2}-8 \pi^{-2} \gamma^{-3} \sqrt{4 \pi^{-4}+\left(\frac{\pi v_{0}}{\Delta x}\right)^{2} \gamma^{3}}\right|} .
$$

The predicted threshold grid spacing compares well to the results obtained from numerically solving Eq. (44) using a "divide and conquer" method to determine the maximum stable grid spacing. This is illustrated in Table 1.

\begin{tabular}{ccc}
\hline \hline$v_{0}$ & $\Delta x_{\text {th }}$ & $\Delta x_{-}$ \\
\hline $1 / 20$ & 0.2746 & 0.2741 \\
$1 / 10$ & 0.5523 & 0.5517 \\
$3 / 20$ & 0.8363 & 0.833 \\
$1 / 5$ & 1.130 & 1.128 \\
$1 / 4$ & 1.438 & 1.435 \\
$1 / 2$ & 3.401 & 3.396 \\
$\sqrt{3} / 2$ & 13.43 & 13.40 \\
$9 / 10$ & 17.14 & 17.12 \\
$95 / 100$ & 29.84 & 29.82 \\
$975 / 1000$ & 51.02 & 50.98 \\
$990 / 1000$ & 579.2 & 578.8 \\
\hline
\end{tabular}

Table 1: Comparison of the analytic and numerically determined threshold grid spacing for the 1-D dispersion relation Eq. (44) when $\Delta t \rightarrow 0$. The maximum grid spacing that does not result in an instability is predicted to be $\Delta x_{\mathrm{th}}$. The largest grid spacing resulting in completely real solutions, found from numerically solving Eq. (44), is given by $\Delta x_{-}$.

\subsection{Instability from the Fully Discrete Electrostatic-like Mode}

The relationship between the parameters $\Delta x$ and $\Delta t$ of Eq. (42) and the numerical instability is investigated in this section for $\Delta t>0$. Because of the discretization in both space and time, all solutions of Eq. (38) for $\omega$ in each spatial and temporal Brillouin zone should be identical. We again define $\omega_{t}=\omega-k v_{0}=\omega_{r}+i \omega_{i}$, and shall focus on the fundamental zone, i.e., the zone located in $\omega_{r} \Delta t \in[-\pi, \pi]$ (note that this is shifted

\footnotetext{
${ }^{6}$ An equation similar to Eq. (45), but with the $q=+1$ term replaced with the $q=-1$ term, can be evaluated at $k \Delta x=-\pi$ to obtain a similar result.
} 
from the fundamental $\omega \Delta t$ Brillouin zone, but that does not affect our analysis since $\omega \Delta t$ Brillouin zones are periodic), and $k \Delta x \in[-\pi, \pi]$. Not surprisingly, the finite $\Delta t$ leads to a significantly more complicated numerical instability analysis, mainly due to the interaction of an $\omega \approx 0$ mode with other modes in the system, and the temporal Brillouin zones that change the location of the alias modes compared to the $\Delta t=0$ case. Multiple instabilities can exist simultaneously for a particular set of parameters, for which we will focus on the leading instability with the largest growth rate. This analysis will show that, unlike in the $\Delta t \rightarrow 0$ case, there is always a non zero growth rate associated with a non zero drift velocity, regardless of time step and grid size.

When examining the behavior of solutions in the fundamental zone, we shall focus our discussion on those originating from terms in Eq. (39) with $q \geq 0$, since solutions from $q \leq 0$ terms are just antisymmetric with these in $k \Delta x$. Note that solutions for $\omega_{r} \Delta t$ to Eq. (38) in neighboring spatial Brillouin zones will be identical up to a phase shift of $\pm 2 \pi v_{0} \Delta t / \Delta x$, dependent on whether the Brillouin zone to the left $(+)$ or right $(-)$ is considered. This is again a consequence of searching for solutions to $\omega_{t}$, instead of $\omega$.

\subsubsection{Approximation of the Dispersion Relation by Truncation}

Evaluating the infinite sum in Eq. (42) exactly is difficult, so instead we multiply Eq. (42) by $\gamma^{3} \csc ^{4}(k \Delta x / 2) /(4 S \Delta x)$, truncate the infinite sum at the $N^{\text {th }}$ term and make the approximation

$$
\epsilon_{a}^{N} \approx 0
$$

with

$$
\begin{aligned}
\epsilon_{a}^{N} & =\frac{\gamma^{3} \csc ^{4}(k \Delta x / 2)}{2 S^{2} \Delta x^{2}} \sin \left[\left(S v_{0} k \Delta x+\omega_{t} \Delta t\right) / 2\right] \\
& -\sum_{q=-N}^{N} \frac{2}{(k \Delta x-2 \pi q)^{4}} \csc \left(\pi q S v_{0}+\omega_{t} \Delta t / 2\right) \\
& -\sum_{q=-N}^{N} \frac{S v_{0}}{(k \Delta x-2 \pi q)^{3}} \csc ^{2}\left(\pi q S v_{0}+\omega_{t} \Delta t / 2\right) \cos \left(\pi q S v_{0}+\omega_{t} \Delta t / 2\right),
\end{aligned}
$$

where $S=\Delta t / \Delta x$ is the Courant number. For the FDTD Yee solver to be stable we require $S<1$.

There are, however, unavoidable effects of approximating the sum in Eq. (42) with any finite sum. One effect is that aliases from truncated terms will not be present. This effect will be most significant when an alias associated with such a term is responsible for the leading, or near leading, instability. However, the leading instability is usually due to the first few terms in the sum, as further terms decrease at least as fast as $1 /(k \Delta x-2 \pi q)^{3}$. This consideration justifies a truncation of the infinite sum at a relatively small $N$. Another unavoidable effect is that solutions across all Brillouin zones will not be symmetric. These effects are artifacts of the approximation and not actually properties of Eq. (42).

Figure 4 shows the convergence of solutions responsible for the leading instability for some parameters of interest. It indicates that, in addition to $\omega_{\mathrm{ES}}^{ \pm}$and $|q|>0$ alias 
(a) $\omega_{r} \Delta t$

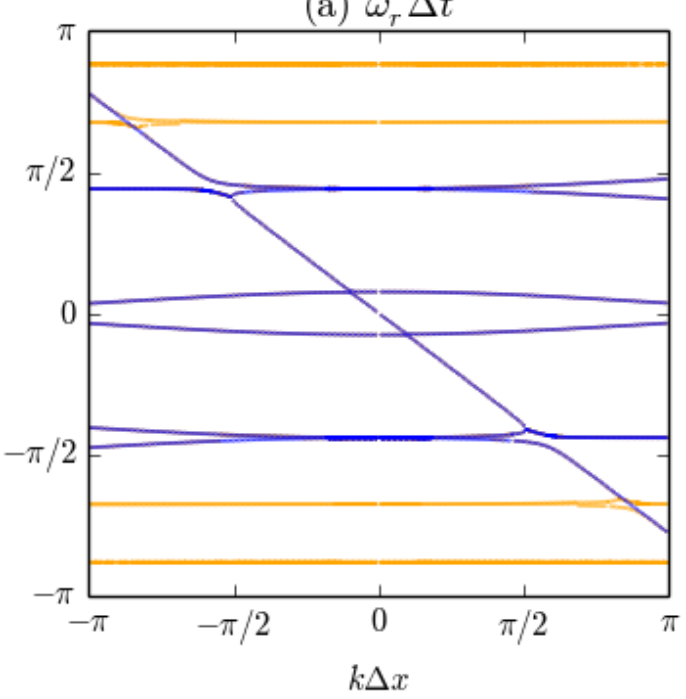

(b) $\omega_{i} \Delta t$

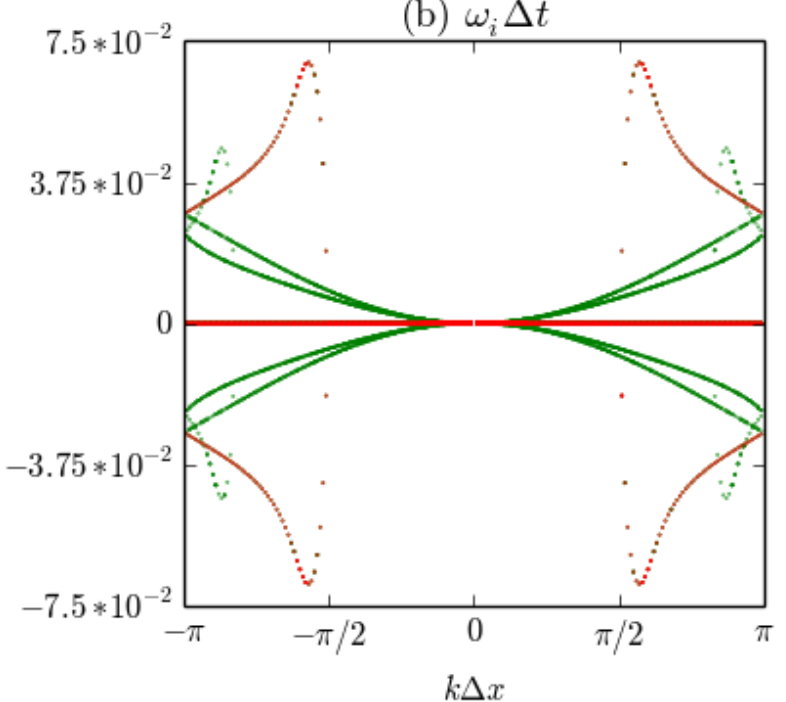

Figure 4: Solutions to Eq. (49) with $N=1$ (Blue, Red) and $N=3$ (Orange, Green) for $S=0.9, v_{0}=\sqrt{3} / 2, \Delta x=0.75$. Plot (a) shows solutions for $\omega_{r} \Delta t$, and plot (b) shows solutions for $\omega_{i} \Delta t$. The orange and green curves are also present "underneath" the blue and red curves.

modes, similar to the $\Delta t \rightarrow 0$ case, there is a negative-slope linear mode that passes through the origin. This mode corresponds to the second $\omega=0$ mode that could not be factored from $\epsilon_{a}$, as was possible in Eq. (44) for the $\Delta t \rightarrow 0$ case. The inability to factor this mode from Eq. (39) stems from the inexact charge conservation in the current deposition scheme of Eq. (6). It can be shown that solutions with $\omega=0$ (exactly) and $\vec{J}=\overrightarrow{0}$ (exactly) only exist if charge is exactly conserved. Therefore, a solution of $\omega \approx 0$ is embedded in $\epsilon_{a}$ in Eq. (42).

This mode is given approximately by $\omega_{t 0} \Delta t \approx-k \Delta x \mathcal{S}$, where we have introduced the scaled Courant number $\mathcal{S}=S v_{0}$. Furthermore, the vertical locations of the $|q|>0$ alias modes are no longer necessarily proportional to $q$ as these modes need to be folded into the fundamental Brillouin zone and can intersect $\omega_{t 0} .{ }^{7}$ With these differences, one can expect an instability to occur in the following two scenarios:

1. The finite space-time E.S.-like mode and alias modes intersect each other. Because we are interested only in the leading instability, we will analyze the intersection of the finite space-time E.S.-like mode and the $q= \pm 1$ aliases in detail and note that this is of concern only over limited parameter ranges.

2. Alias modes intersect $\omega_{t 0}$ (see Figure 4). This causes instability across a significant portion of the parameter ranges and is often responsible for the leading instability.

${ }^{7}$ The modes are "folded" because solutions are periodic in Fourier space. This is true of any discrete system, and is not a consequence of sampling. Aliasing due to sampling involves an infinite sum over all Brillouin zones and is different from folding the modes. 
To investigate these two types of instabilities, we will first determine the location of the $|q|>0$ alias modes. Similar to the $\Delta t \rightarrow 0$ case, in the non-relativistic limit (i.e. $\left.v_{0} \ll 1\right)$ and for sufficiently small $q$, the location of the $q^{\text {th }}(q \neq 0)$ alias is determined by $\omega_{t}^{q}=-2 \pi q v_{0} / \Delta x$ or $\omega_{t}^{q} \Delta t=-2 \pi q \mathcal{S}$. The relativistic generalization can be found from the pole, $\sin \left(\pi q \mathcal{S}+\omega_{t}^{q} \Delta t / 2\right)=0$, in the $q^{\text {th }}$ term in Eq. (49). The solution is $\omega_{t}^{q} \Delta t=2 \pi(n-q \mathcal{S})$, where $n$ is an integer and $n \in[q \mathcal{S}-1 / 2, q \mathcal{S}+1 / 2)$ for $q>0$, $n \in(q \mathcal{S}-1 / 2, q \mathcal{S}+1 / 2]$ for $q<0$, so $\omega_{t}^{q} \Delta t \in[-\pi, \pi]$. Note that, in contrast to the non-relativistic case, it is possible for the value of an alias to switch signs (i.e., for some $\mathcal{S}$ we can have $\omega_{t}^{+|q|} \Delta t>0$, and $\left.\omega_{t}^{-|q|} \Delta t<0\right)$.

To ensure that an intersection of the second type will occur, we must enforce that the aliases with $q>0(q<0)$ span at least part of the $\omega_{t} \Delta t$ range occupied by the positive (negative) segment of $\omega_{t 0}$ (i.e., at least one of these aliases must intersect $\omega_{t 0}$ ). The spacing between consecutive aliases is $2 \pi \mathcal{S}$. Therefore, the condition on $\mathcal{S}$ and $N$ is $2 \pi N \mathcal{S}+\pi \mathcal{S}>2 \pi$, or $\mathcal{S}>1 /(N+1 / 2)$. For the two $N$ values used to obtain the plots in Figure 4, this condition is satisfied by $N=1$. We see the mode responsible for the leading instability does not change, nor does the magnitude of the leading instability when we set $N=3$.

The locations of the alias modes deserve some further discussion. When $2 \mathcal{S}=l / \mathrm{m}$, where $l, m$ are coprime integers, it can be shown that there are only $2 m+1$ possible locations equally spaced in the interval $\omega_{t} \Delta t \in[-\pi, \pi]$ for the alias modes. For example, when $\mathcal{S}=1 / 2$, all $q>0$ aliases will be located at $\omega_{t}^{q} \Delta t=\{-\pi, 0\}$ (although the exact location of the alias branches may be somewhat different than these two values). The $q<$ 0 modes will be at symmetric locations between 0 and $\pi$. Similarly, for $\mathcal{S}=\{1 / 4,3 / 4\}$, we find $\omega_{t}^{q} \Delta t=\{0, \pm \pi / 2, \pm \pi\}$. No instability due to alias-alias interactions is observed for these exact $\mathcal{S}$ values with typical $\Delta x \lesssim 1$, and this is illustrated in Figure 5 . The instability in Figure 5 (b) is of the second kind. It is also worth noting that when an alias mode is located on the $k \Delta x$-axis, no instability of the first or second kind is associated with that alias. For other $\mathcal{S}$ values, although many aliases may overlap, aliasalias interactions will not be responsible for the leading instability. This is because the indices $q_{1}$ and $q_{2}$ of two different alias modes having the same location satisfy $\left|q_{1}-q_{2}\right|=$ $\left|\left(n_{1}-n_{2}\right) / \mathcal{S}\right| \geq \mathcal{S}^{-1}$. For $\mathcal{S} \ll 1$ and $\left|q_{1}-q_{2}\right| \gg 1$, therefore, this type of intersection involves a high $|q|$ alias mode and the contribution of such a mode in the infinite sum is always suppressed.

\subsubsection{Instability from $\omega_{\mathrm{ES}}^{-}$and the $q=1$ Alias}

In the case that $\mathcal{S}$ is sufficiently small, the $q=1$ alias is near $\omega_{\mathrm{ES}}^{-}$. It also has branches extending above and below $\omega_{t}^{1} \Delta t$. The existence of these two branches can be understood by recalling that solutions to Eq. (38) in each spatial and temporal Brillouin zone should be identical (up to a $2 \pi v_{0} / \Delta x$ shift in $\omega_{t}$, or a $2 \pi \mathcal{S}$ shift in $\omega_{t} \Delta t$ ). In the spatial Brillouin zone immediately to the right of the fundamental zone, the $q=1$ alias is identical in form to the finite space-time E.S.-like mode in the fundamental zone. Therefore, one can calculate the branch separation of the $q=1$ alias at $k \Delta x=\pi$ by the branch separation of the finite space-time E.S.-like mode at $k \Delta x=\pi$.

The branch separation of the finite space-time E.S.-like mode at $k \Delta x=\{0, \pm \pi\}$ can 
(a) $\omega_{r} \Delta t$

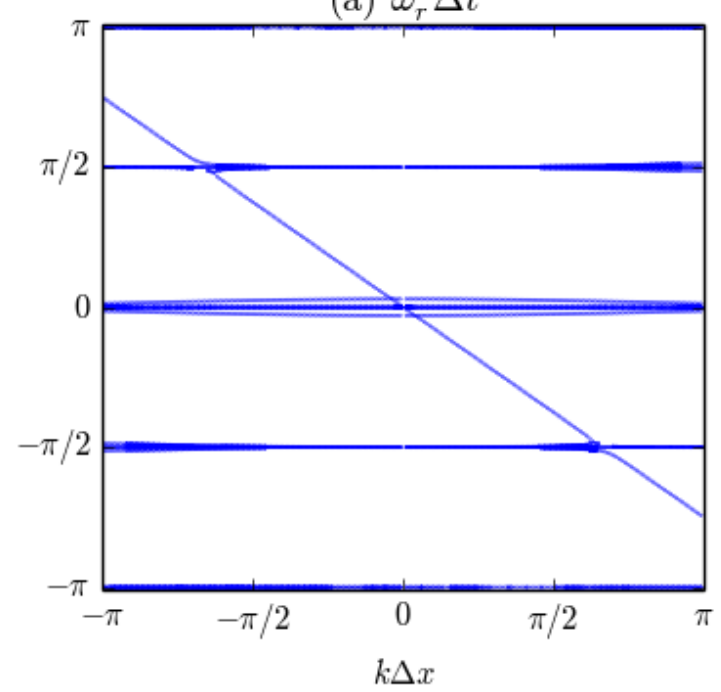

(b) $\omega_{i} \Delta t$

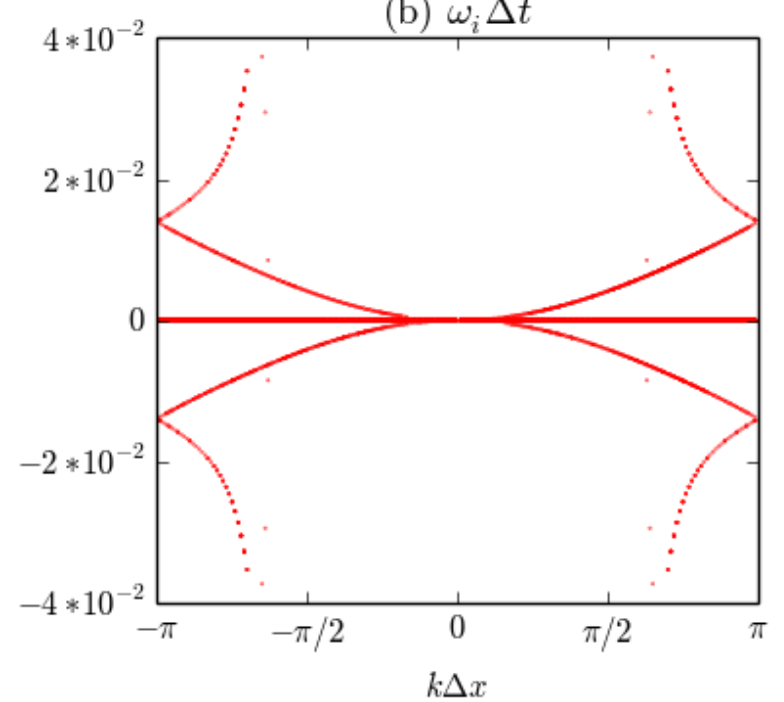

Figure 5: Numerical solutions to Eq. (49) with $N=4$ for $\mathcal{S}=0.75\left(S=0.9, v_{0}=5 / 6\right)$ and $\Delta x=0.25$. The locations of the aliases in (a) are $q= \pm 1 \rightarrow \pm \pi / 2, \pm 2 \rightarrow \pm \pi$, $\pm 3 \rightarrow \mp \pi / 2$, and $\pm 4 \rightarrow 0$.

be found by including only the $q=0$ term from the sum in Eq. (49). At $k \Delta x=0$, we obtain

$$
2 \gamma^{3}\left[\cos \left(\omega_{t} \Delta t\right)-1\right]+S^{2} \Delta x^{2}=0,
$$

which implies the finite space-time E.S.-like branches cross the $\omega_{t} \Delta t$ axis at $\omega_{\mathrm{ES}}^{ \pm} \Delta t(k \Delta x=$ $0)= \pm \arccos \left(1-S^{2} \Delta x^{2} / 2 \gamma^{3}\right)$. Therefore, the branch separation of this mode at $k \Delta x=$ 0 is $2 \arccos \left(1-S^{2} \Delta x^{2} / 2 \gamma^{3}\right)$. When $S \Delta x / \gamma^{3 / 2} \ll 1$, we have $\omega_{\mathrm{ES}}^{ \pm} \Delta t(k \Delta x=0) \approx$ $\pm S \Delta x / \gamma^{3 / 2}= \pm \Delta t / \gamma^{3 / 2}$ and the branch separation is about $2 S \Delta x / \gamma^{3 / 2}=2 \Delta t / \gamma^{3 / 2}$.

At $k \Delta x=\pi$, we obtain

$$
\frac{\gamma^{3} \sin \left[\left(\pi S v_{0}+\omega_{t} \Delta t\right) / 2\right]}{2 S^{2} \Delta x^{2}}-\frac{2 \csc \left(\omega_{t} \Delta t / 2\right)}{\pi^{4}}-\frac{S v_{0} \cos \left(\omega_{t} \Delta t\right) \csc ^{2}\left(\omega_{t} \Delta t\right)}{\pi^{3}}=0,
$$

which can be solved analytically to give $\omega_{\mathrm{ES}}^{ \pm} \Delta t(k \Delta x=\pi)$ and the branch separation there. The result is again very complicated and not given here. Instead we present an approximate yet simple prediction of the instability from $\omega_{\mathrm{ES}}^{ \pm}$and the $q= \pm 1$ aliases below.

Similar to the $\Delta t \rightarrow 0$ case, when the upper (lower) branch of the $q=1(q=-1)$ alias mode and $\omega_{\mathrm{ES}}^{-}\left(\omega_{\mathrm{ES}}^{+}\right)$intersect at $k \Delta x=\pi(-\pi)$, it causes an instability, as shown in Figure 6. In Figure 6 (b), the upper lobe near $k \Delta x=\pi$ is associated with the upper branch of the $q=1$ alias, and the lower lobe is from $\omega_{\mathrm{ES}}^{-}$.

The condition for these two modes to "touch" at $k \Delta x=\pi$ can be approximately found by letting $\left|\omega_{\mathrm{ES}}^{-} \Delta t(k \Delta x=0)\right|=\left|\omega_{t}^{1} \Delta t(k \Delta x=0)\right|$, i.e., $\Delta t / \gamma^{3 / 2}=2 \pi \mathcal{S}$, which gives $\Delta x_{\mathrm{th}}^{\delta}=2 \pi v_{0} \gamma^{3 / 2}$, where the superscript $\delta$ is used to denote this close encounter type of mode intersection. When $\Delta x$ is close to or larger than this value, the intersection of the 

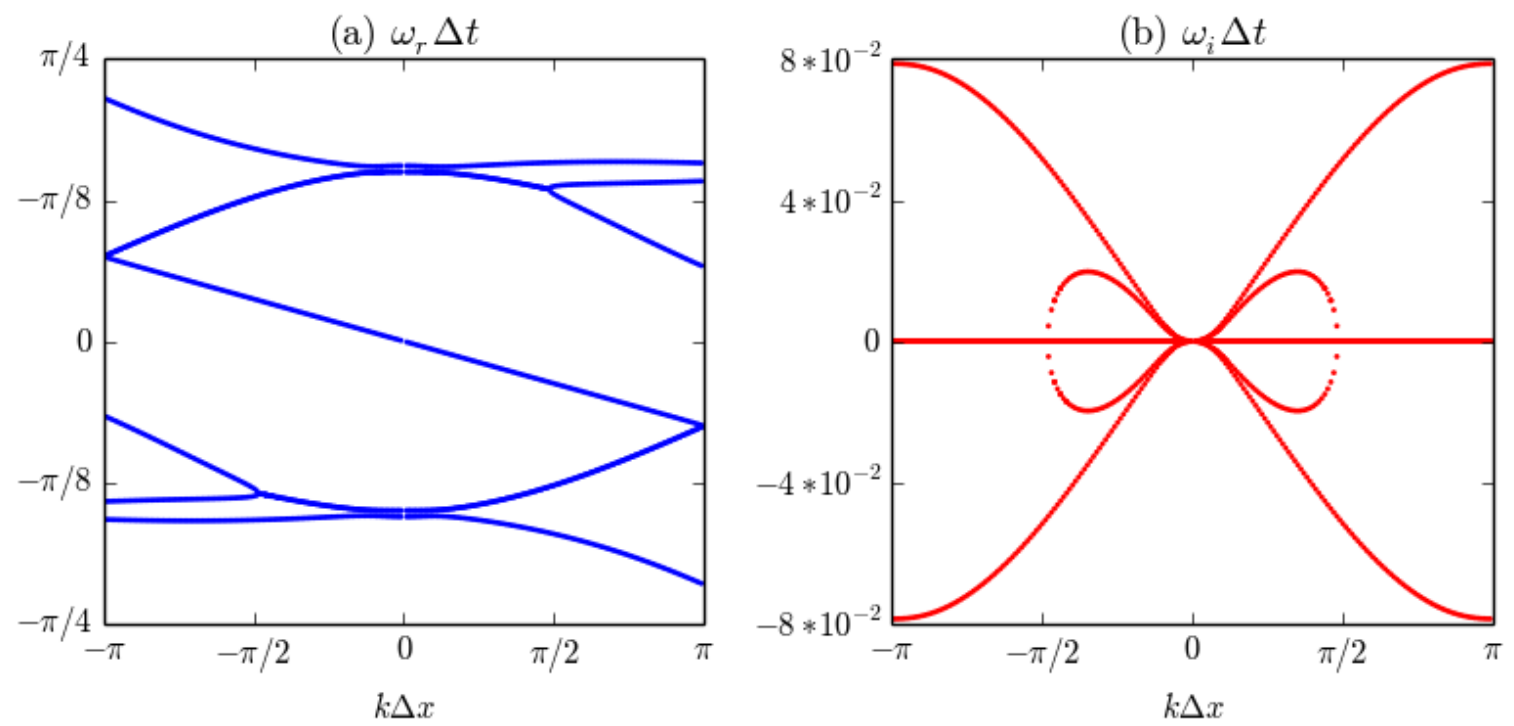

Figure 6: The $\delta$-instability illustrated by solving Eq. (49) with $N=1, \mathcal{S}=0.075$, $\Delta x=0.65$, and $v_{0}=0.1$. Panel (a) shows $\omega_{r} \Delta t$, and (b) shows $\omega_{i} \Delta t$. For these parameter values $\Delta x_{\mathrm{th}}^{\delta} \approx 0.6331$.

finite space-time E.S.-like mode and the $q= \pm 1$ aliases gives rise to the instability shown in Figure 6, which we name the $\delta$-instability. The leading instability is, indeed, largest at $k \Delta x= \pm \pi$. The smaller, secondary, instability seen in Figure 6 is also due to the intersection of these modes, but never leads. For $\Delta x \lesssim \Delta x_{\mathrm{th}}^{\delta}$, these modes detach and are stable.

However, due to the folding of high $|q|$ alias modes into a smaller $\left|\omega_{t} \Delta t\right|$ location than the $q= \pm 1$ aliases, it is possible that such a mode can interact with the the finite spacetime E.S.-like mode causing an instability. The growth rate for the instability in this case usually does not lead as it involves a high $|q|$ mode. The threshold of the $\delta$-instability is not very restrictive as typical simulation grid sizes, which must be small enough to resolve parameters like the plasma skin depth, or Debye length, satisfy $\Delta x<\Delta x_{\text {th }}^{\delta}$ for sufficiently large $v_{0}$. However, as $\Delta x$ decreases, the $q= \pm 1$ modes will be folded into the opposite half of the fundamental Brillouin zone and intersect with $\omega_{t 0}$ which is discussed in the next section.

\subsubsection{Instability from the Interaction Between $\omega_{t 0}$ and the Alias Modes}

It can be shown that $\omega_{\mathrm{ES}}^{-}$and the $q=1$ alias first intersect at $k \Delta x=\pi$ and $\omega_{t} \Delta t=-\pi \mathcal{S}$ as the separation of the branches of each mode are equal at $k \Delta x=\pi$. We note that $k \Delta x=\pi$ and $\omega_{t} \Delta t=-\pi \mathcal{S}$ is also on $\omega_{t 0}$, i.e., $\omega_{t 0}$ also intersects $\omega_{\mathrm{ES}}^{-}$and the $q=1$ alias at the same location. It has been observed that the intersection of $\omega_{t 0}$ with the finite space-time E.S.-like mode is always stable, while the intersection with $|q|>0$ alias modes is always unstable.

We devote the remainder of this section to analyzing the instability resulting from 
$\omega_{t 0}$-alias intersections, since this type of instability is by far the most prevalent in the parameter range $v_{0} \lesssim 1$, which is of particular interest.

To obtain a simple expression for the peak growth rate that results from an intersection of the $q^{\text {th }}$ alias with $\omega_{t 0}$, we will approximate the solution to Eq. (38) in the vicinity of $\omega_{t}^{q} \Delta t$ by including only the $q^{\text {th }}$ term in the sum. We can solve

$$
\begin{gathered}
\frac{\gamma^{3} \csc ^{4}(k \Delta x / 2)}{2 S^{2} \Delta x^{2}} \sin \left\{\left(S v_{0} k \Delta x+\omega_{t} \Delta t\right) / 2\right\}-\frac{2}{(k \Delta x-2 \pi q)^{4}} \csc \left(\pi q S v_{0}+\omega_{t} \Delta t / 2\right) \\
-\frac{S v_{0}}{(k \Delta x-2 \pi q)^{3}} \csc ^{2}\left(\pi q S v_{0}+\omega_{t} \Delta t / 2\right) \cos \left(\pi q S v_{0}+\omega_{t} \Delta t / 2\right)=0
\end{gathered}
$$

exactly for $\omega_{t} \Delta t$.

The analytic solutions to Eq. (52) shown in Figure 7 are accurate for all $k \Delta x$, as long as there is no overlapping with a smaller $|q|$ index alias. These solutions are also complicated. We focus on the $q>0$ alias and notice that the peak growth rate of this alias occurs near where it intersects $\omega_{t 0}$. We then solve Eq. (52) with $k \Delta x=2 \pi(q \mathcal{S}-n) / \mathcal{S}$, where $n$ is an integer and $q \mathcal{S}-1 / 2 \leq n<q \mathcal{S}+1 / 2$. When $n=0$, the $q^{\text {th }}$ alias is at $\omega \Delta t=-2 \pi q \mathcal{S}$ and not folded into the upper half of the fundamental Brillouin zone, while $\omega_{t 0} \in[-\pi \mathcal{S}, \pi \mathcal{S}]$. Therefore, there is no interaction or instability between $\omega_{t 0}$ and alias modes for $n=0$ (i.e., when this mode is not folded).

For $n \neq 0$, one can rewrite Eq. (52) as

$$
1+c_{1} \cot (\alpha)+c_{2} /\left[1+\cot ^{2}(\alpha)\right]=0,
$$

where $c_{1}=-n \pi, c_{2}=4(-1)^{n+1} \gamma^{3}\left[S \Delta x \operatorname{sinc}^{2}(n \pi / \mathcal{S})\right]^{-2}$, and $\alpha=\pi q \mathcal{S}+\omega_{t} \Delta t / 2$. Since $n \neq 0$, the $q^{\text {th }}$ alias is folded and there are three solutions to Eq. (53) in the fundamental Brillouin zone. One solution for $\omega_{t} \Delta t$ is completely real, and two solutions have a nonzero imaginary part. The latter are of interest and are complex conjugates.

For $\left|\omega_{i} \Delta t\right| \ll 1$, we find that

$$
\omega_{i} \Delta t \approx \frac{(-1)^{n+1} \sqrt{3}(n \pi)^{1 / 3}}{\gamma}\left[\operatorname{sinc}^{2}(n \pi / \mathcal{S}) S \Delta x / 2\right]^{2 / 3} .
$$

Equation (54) allows us to predict the growth rate at the intersection of the $q^{\text {th }}$ alias with $\omega_{t 0}$, when $q \mathcal{S} \in(n-1 / 2, n+1 / 2]$.

For the $q<0$ alias modes, we solve an equation that is exactly the same as Eq. (53) to get the same peak growth rate from their intersections with $\omega_{t 0}$. This is due to the symmetry of $\pm q$ alias solutions. We thus conclude that Eq. (54) gives the peak growth rate for the intersection of the $q^{\text {th }}$ alias with $\omega_{t 0}$ for $|q|>0$. Table 2 compares the instability predicted by Eq. (54) to that obtained by solving Eq. (49) at the intersection point.

\section{Simulation Verification}

Simulations to verify the validity of solutions to Eq. (42) and the expression for the growth rate due to an $\omega_{t 0}$-alias intersection given in Eq. (54) have been carried out using 
(a) $\omega_{r} \Delta t$

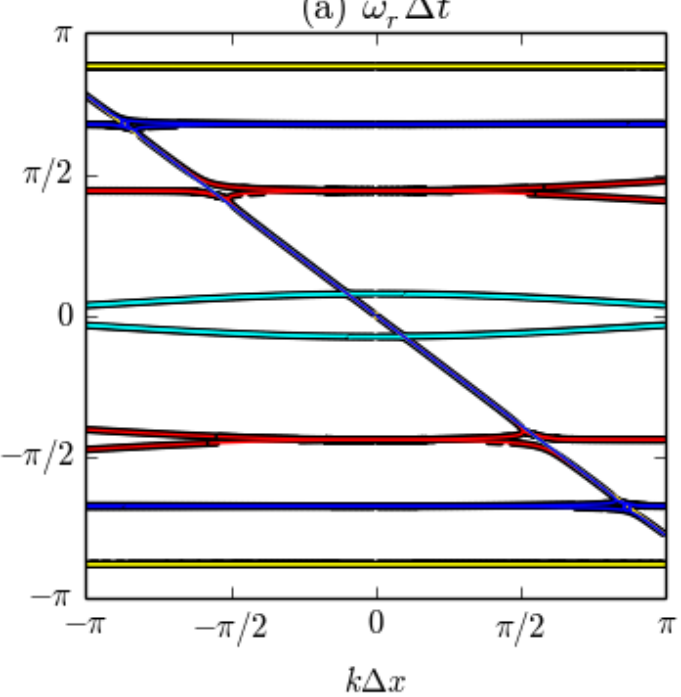

(b) $\omega_{i} \Delta t$

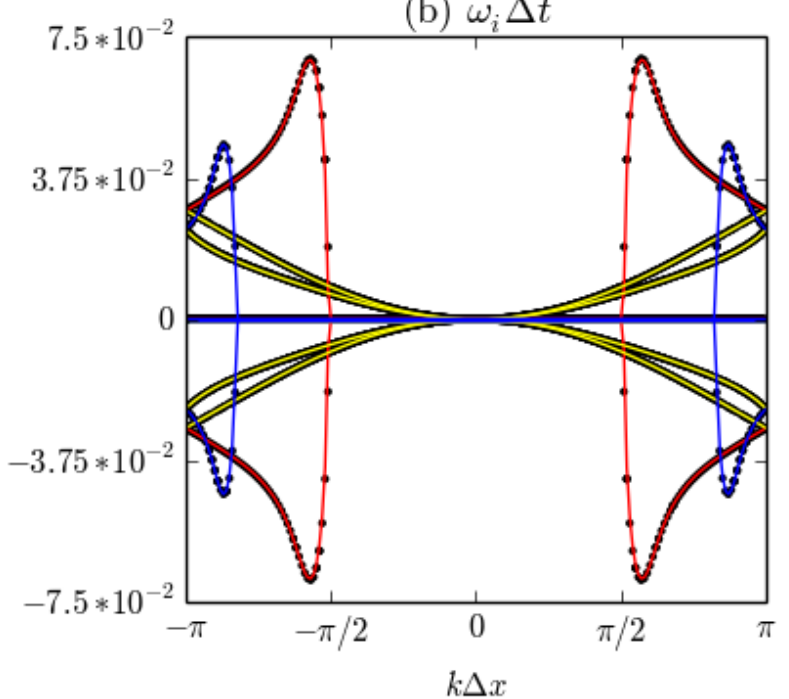

Figure 7: Approximated analytic solutions (solid lines) to Eq. (52) for the finite spacetime E.S.-like mode (cyan), and the $q= \pm 1 \rightarrow \pm 1.386$ (red), $\pm 2 \rightarrow \pm 2.772$ (yellow), $\pm 3 \rightarrow \mp 2.125$ (blue) aliases. They are overlaid on the numerical solutions (scatter) to Eq. (49) with $N=3$ for $S=0.9, v_{0}=\sqrt{3} / 2, \Delta x=0.75$. The numbers to the right of the arrows give the alias locations. Panel (a) shows solutions for $\omega_{r} \Delta t$, and (b) shows solutions for $\omega_{i} \Delta t$.

the E.M. PIC code OSIRIS [21] [22]. Figure 8 shows the dispersion plot and growth rates for the parameters of Figure 4.

The simulation was initialized with 1000 electrons and 1000 free streaming protons per cell. The simulation box was set to 1024 cells with periodic boundary conditions. The perturbation was seeded with an electric field of $E_{x}(x, t=0)=2 * 10^{-10} \sin \left(k_{1} x\right)$ in normalized simulation units, where $k_{1}$ is the wavenumber where $\omega_{t 0}$ intersects the $q=1$ alias. We also note that seeding the same field at $k_{2}$ (where the $q=2$ alias is intersected) produces very similar results.

The dispersion plot and growth rates in Figure 8 were obtained from time steps 50 to 200 , i.e., during the period of exponential growth, and in the linear regime. The growth rates were calculated by fitting a line to $\log \left(\left|E_{x}(k \Delta x, t)\right|\right)$ for each Fourier mode. Figure 8 (b) shows the simulation is in very good agreement with the analytic solutions for the fastest growing modes.

\section{Discussion}

\subsection{A Comparison of Results with Previous Treatments}

The results of Reference [14] are the most immediately comparable to the results given in this paper. The significant difference in the forms of the current (our Eq. (34) vs. the 


\begin{tabular}{ccccc}
\hline \hline$q$ & $\omega_{i} \Delta t_{\text {pred }}$ & $\omega_{i} \Delta t_{\text {solved }}$ & $\omega_{i} \Delta t_{\text {pred }}$ & $\omega_{i} \Delta t_{\text {solved }}$ \\
\hline 1 & 0.0684 & 0.0692 & 0.0000 & 0.0000 \\
2 & 0.0466 & 0.0460 & 0.0045 & 0.0048 \\
3 & 0.0466 & 0.0465 & 0.0045 & 0.0048 \\
4 & 0.0112 & 0.0115 & 0.0045 & 0.0048 \\
5 & 0.0071 & 0.0073 & 0.0045 & 0.0048 \\
6 & 0.0183 & 0.0185 & 0.0049 & 0.0049 \\
7 & 0.0183 & 0.0185 & 0.0049 & 0.0049 \\
8 & 0.0121 & 0.0062 & 0.0049 & 0.0049 \\
9 & 0.0003 & 0.0002 & 0.0049 & 0.0049 \\
10 & 0.0080 & 0.0000 & 0.0042 & 0.0042 \\
\hline
\end{tabular}

Table 2: The growth rates predicted by Eq. (54), $\omega_{i} \Delta t_{\text {pred }}$, compared to the growth rates found by solving Eq. (49) with $N=10$ at the alias- $\omega_{t 0}$ mode intersection point, $\omega_{i} \Delta t_{\text {solved }}$. The left two columns are for $S=0.9, \Delta x=0.75$, and the right two columns are for $S=0.3, \Delta x=1$. Both cases are for $v_{0}=\sqrt{3} / 2$. The top entries in the right two columns are associated with $n=0$ from Eq. (54); no instability is predicted, nor is any found from solving Eq. (49). The slight discrepancy in growth rates of high $|q|$ aliases in the first two columns is attributed to their close vicinity to lower $|q|$ value aliases.

right hand side of Eq. (13) of Reference [14]) is attributed exclusively to the assumption of temporal aliasing in Reference [14]. Even so, upon evaluating the sum over the temporal aliases in that reference's Eq. (13), one obtains a formula for $\tilde{\vec{J}}$ that is our Eq. (34). The authors of that reference note that they have chosen to absorb the phase factors $\tau_{E}, \tau_{B}$, $\overleftrightarrow{O_{E}}$, and $\overleftrightarrow{O_{B}}$ into their interpolation functions, where we have written ours explicitly.

The 1-D cold plasma dispersion tensor elements in Eqs. (39) and (40) of this paper are the expressions analogous to those in Eq. (19) of Reference [14]. Our results agree exactly with that reference.

Related work was also presented in Reference [13]. Here, however, the Esirkepov charge conserving current deposit scheme [17] is assumed. If the procedure in Section 4 is followed to obtain the current, one arrives at an expression that is identical in form to Eq. (34). The difference is that instead of being given by $\vec{v}=\vec{p} / \gamma$, the charge conserving Esirkepov "velocity" is now

$$
\vec{v}_{\mathrm{E}}=\left(\begin{array}{l}
\frac{\sin \left(k_{q}^{x} v_{x} \Delta t / 2\right)}{k_{B}\left(k_{q}^{x}\right) \Delta t / 2}\left[\cos \left(k_{q}^{y} v_{y} \Delta t / 2\right) \cos \left(k_{q}^{z} v_{z} \Delta t / 2\right)-\frac{1}{3} \sin \left(k_{q}^{y} v_{y} \Delta t / 2\right) \sin \left(k_{q}^{z} v_{z} \Delta t / 2\right)\right] \\
\frac{\sin \left(k_{q}^{y} v_{y} \Delta t / 2\right)}{k_{B}\left(k_{q}^{y}\right) \Delta t / 2}\left[\cos \left(k_{q}^{z} v_{z} \Delta t / 2\right) \cos \left(k_{q}^{x} v_{x} \Delta t / 2\right)-\frac{1}{3} \sin \left(k_{q}^{z} v_{z} \Delta t / 2\right) \sin \left(k_{q}^{x} v_{x} \Delta t / 2\right)\right] \\
\frac{\sin \left(k_{q}^{z} v_{z} \Delta t / 2\right)}{k_{B}\left(k_{q}^{z}\right) \Delta t / 2}\left[\cos \left(k_{q}^{x} v_{x} \Delta t / 2\right) \cos \left(k_{q}^{y} v_{y} \Delta t / 2\right)-\frac{1}{3} \sin \left(k_{q}^{x} v_{x} \Delta t / 2\right) \sin \left(k_{q}^{y} v_{y} \Delta t / 2\right)\right]
\end{array}\right),
$$

which is consistent with Eq. (7) from Reference [13]. The operator $k_{B}$ is the component of the operator $\vec{k}_{B}$ implied by its argument. The expression for the charge conserving current (obtained by replacing $\vec{p} / \gamma$ inside the integral in Eq. (34)) is identical to Eq. 

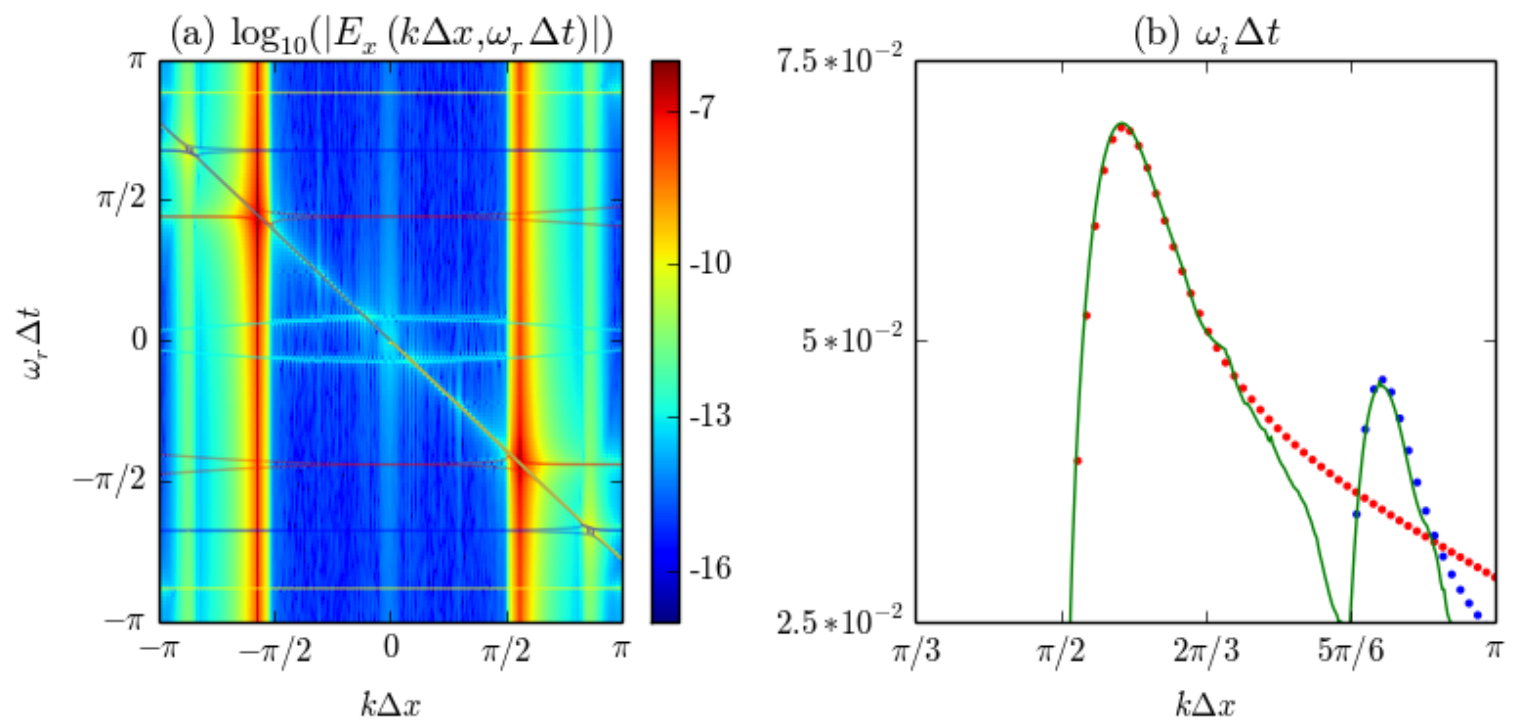

Figure 8: Simulation results for a 1-D drifting cold plasma for the same parameters as Figure 4. Panel (a) shows the dispersion plot for the electric field parallel to the plasma drift from time steps 50 to 200. Analytic solutions from Figure 7 (with the same color labeling) have been overlaid for comparison. Panel (b) shows the growth rate over the same time interval as in (a) for spatial Fourier modes in the vicinity of where $\omega_{t 0}$ intersects the $q=-1$ and $q=3$ aliases. The simulation results are the solid green line, and analytic solutions from Figure 7 have been overlaid in red and blue (scatter), for the $q=-1$ and $q=3$ aliases, respectively.

(10) of Reference [13]. Another widely used charge conserving current deposit scheme is that from Reference [16], for which the charge conserving Villasenor-Buneman "velocity" is given by

$$
\vec{v}_{\mathrm{VB}}=\left(\begin{array}{l}
\frac{\sin \left(k_{q}^{x} v_{x} \Delta t / 2\right)}{k_{B}\left(k_{q}^{x}\right) \Delta t / 2} \operatorname{sinc}\left\{\left(k_{q}^{y} v_{y}+k_{q}^{z} v_{z}\right) \Delta t / 2\right\} \\
\frac{\sin \left(k_{q}^{y} v_{y} \Delta t / 2\right)}{k_{B}\left(k_{q}^{y}\right) \Delta t / 2} \operatorname{sinc}\left\{\left(k_{q}^{z} v_{z}+k_{q}^{x} v_{x}\right) \Delta t / 2\right\} \\
\frac{\sin \left(k_{q}^{z} v_{z} \Delta t / 2\right)}{k_{B}\left(k_{q}^{z}\right) \Delta t / 2} \operatorname{sinc}\left\{\left(k_{q}^{x} v_{x}+k_{q}^{y} v_{y}\right) \Delta t / 2\right\}
\end{array}\right) .
$$

In the $1-\mathrm{D}$ case, $\vec{v}_{\mathrm{E}}$ and $\vec{v}_{\mathrm{VB}}$ are identical. Then, the dispersion relation on the Yee mesh is

$\sin \left\{\left(S v_{0} k \Delta x+\omega_{t} \Delta t\right) / 2\right\}\left[1-\frac{2 S^{2} \Delta x^{2} \sin ^{3}(k \Delta x / 2)}{\gamma^{3}} \sum_{q=-\infty}^{\infty}(-1)^{q} \frac{\csc ^{2}\left(\pi q S v_{0}+\omega_{t} \Delta t / 2\right)}{(k \Delta x-2 \pi q)^{3}}\right]=0$.

This is the same as the result one obtains using Eqs. (7), (9), and (10), the interpolation functions, and Fourier space finite difference operators from Reference [13] to construct the current.

The first factor in Eq. (57) has a solution of $\omega_{t 0}$, which is detached completely from the aliases. Instead, the aliases are unstable all by themselves. Numerical solutions to 
Eq. (57) with the same parameters as Figure 4 and $q=-2$ to 2 are shown in Figure 9 (a) and (b), and simulation results showing the dispersion plot and growth rates are in (c) and (d).

(a) $\omega_{r} \Delta t$

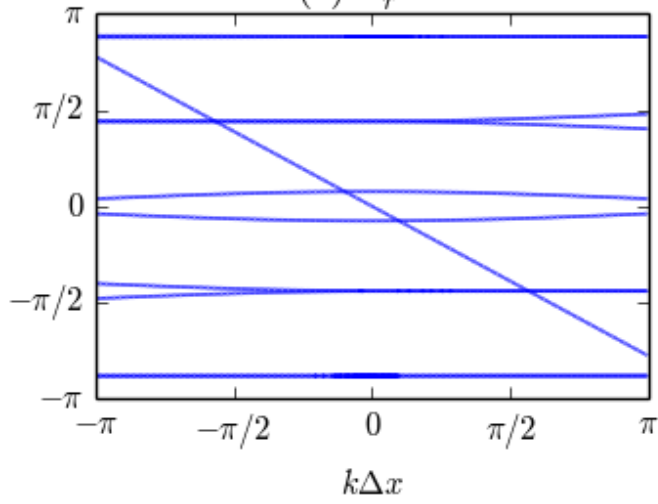

(c) $\log _{10}\left(\left|E_{x}\left(k \Delta x, \omega_{r} \Delta t\right)\right|\right)$

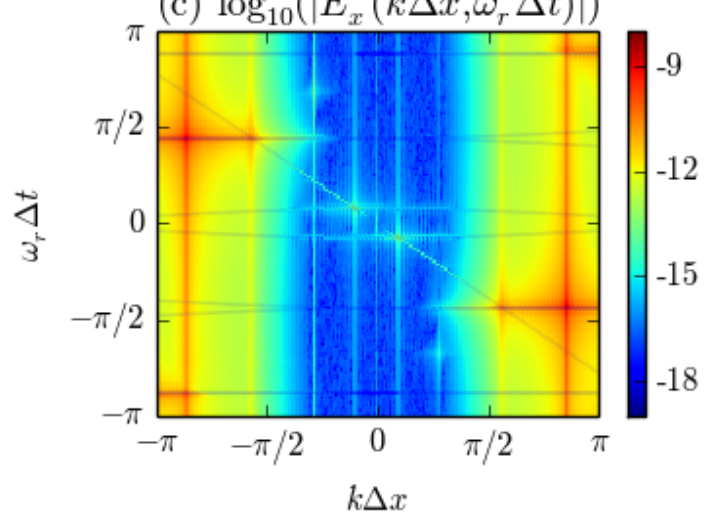

(b) $\omega_{i} \Delta t$

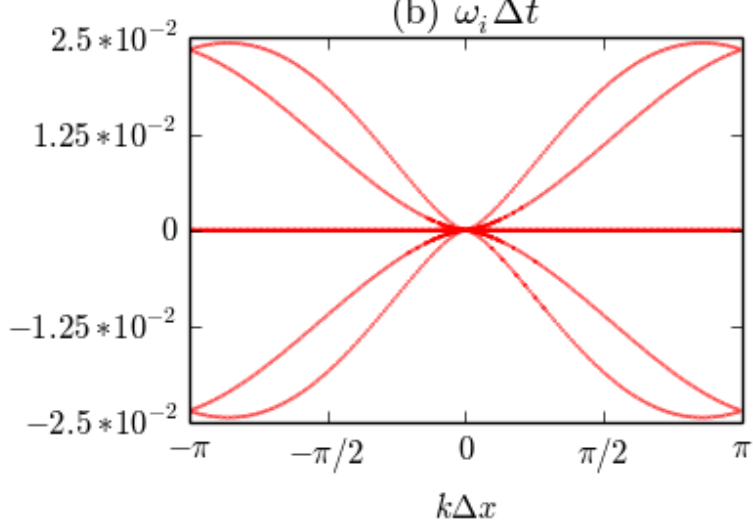

(d) $\omega_{i} \Delta t$

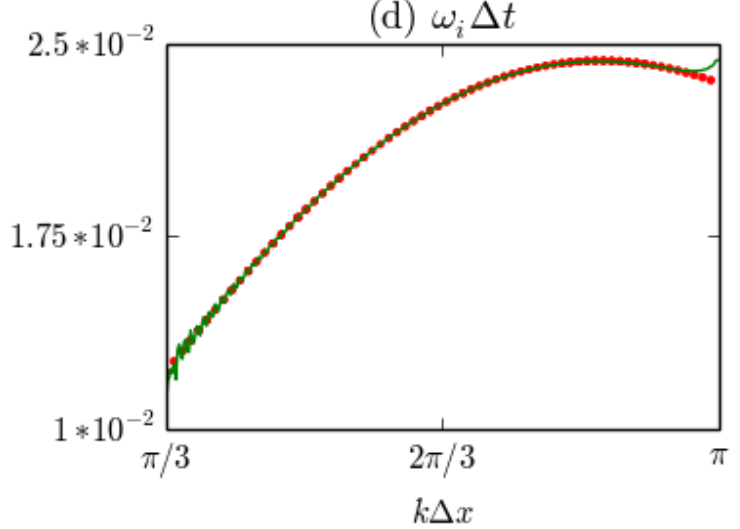

Figure 9: Numerical solutions to Eq. (57) for the same parameters as in Figure 4, with $q=-2$ to 2 , and the simulation results that verify them. Panels (a) and (b) are the numerical solutions for the real and imaginary parts of $\omega_{t} \Delta t$. The positions of the aliases are $q= \pm 1 \rightarrow \pm 1.386, \pm 2 \rightarrow \pm 2.772$. The outer (larger) lobes near $k \Delta x= \pm \pi$ are associated with the $q=\mp 1$ aliases, and the inner (smaller) lobes belong to the $q= \pm 2$ aliases. Panel (c) is the dispersion plot from time steps 350 to 500, and the numerical solutions from (a) have been overlaid for comparison. Panel (d) shows the growth rate over the same time interval as in (c) for spatial Fourier modes in the vicinity of the fastest growing mode. The simulation results are the solid green line, and numerical solutions for the fastest growing mode in (b), from the $q=-1$ alias, have been overlaid in red (scatter).

This simulation was initialized with the same particle parameters, box size, boundary conditions, and initial field amplitude as in the run presented in Section 6. The only difference is that here the perturbing wave number was set to $k=3.6$, which is near where the numerical solutions predict the most unstable mode should be. The simulation dispersion plot and growth rates were obtained from time steps 350 to 500. The growth 
rates were obtained using the same method as in Section 6.

A comparable treatment for the dispersion relation with an infinitesimal time step, Eq. (44), comes from chapter 8-11 of Reference [1]. Equation (7) of chapter 8-11 describes the non-relativistic $(\gamma \rightarrow 1)$ E.S. mode with an infinitesimal time step derived for an E.S. $\mathrm{PIC}$ code. The dispersion relation is

$$
1-4 \Delta x^{2} \sin ^{2}(k \Delta x / 2) \sin (k \Delta x) \sum_{q=-\infty}^{\infty} \frac{1}{(k \Delta x-2 \pi q)^{3}\left(\omega_{t} \Delta x+2 \pi q v_{0}\right)^{2}}=0 .
$$

Solving Eq. (58) with, for example, $v_{0}=0.1$ and $\Delta x=0.5$, reveals that the aliases are unstable by themselves without intersecting. This stands in contrast to the findings of Section 5 and Figure 2 (a) and (b).

\subsection{Consequences of Not Conserving Charge}

There are two types of charge conservation in PIC. They stem from the differential (microscopic) and integral (macroscopic) forms of the continuity equation, respectively. The simple, direct current deposit does not satisfy either of these forms. The most notable consequence for the analysis of the finite grid instability is the interaction of the $\omega_{t 0}$ mode with the alias modes, and the stability of the alias modes.

Recall that the $\omega_{t 0}$ mode corresponds to $\omega \approx 0$. From Eq. (39), we see that $\omega=0$ is an exact solution to Eq. (42) if and only if $\tilde{J}=0$. In $1-\mathrm{D}$, the numerical continuity equation in Fourier space is

$$
\frac{\sin (k \Delta x / 2)}{(\Delta x / 2)} \tilde{J}-\frac{\sin (\omega \Delta t / 2)}{(\Delta t / 2)} \tilde{\rho}=0 .
$$

We see that this is satisfied, exactly, for non trivial $k$ and $\tilde{\rho}$ (i.e., a non zero, propagating, perturbation), when $\omega=0$ and $\tilde{J}=0$ simultaneously. That is, an exact solution of $\omega=0$ to Eq. (42) coincides with exact charge conservation. If we do not require Eq. (59) to be true for our model, then we cannot expect such a model to support an $\omega=0$ mode.

For models which do enforce microscopic charge conservation, e.g., 1-D models employing the Esirkepov or Villasenor-Buneman schemes, we find that the $\omega_{t 0}$ mode does detach from the alias modes. In this case, the leading instability is not caused by that intersection, but does not obey the growth rate predicted by Eq. (48), or threshold grid sizes of Eq. (47) or $\Delta x_{\mathrm{th}}^{\delta}$.

\section{Summary}

The PIC method is particularly useful for studying the kinetic behavior of plasma, due to its first principles treatment of the particle and field dynamics, and self-consistent solution. Increasingly, PIC models, especially E.M. PIC models, are being applied to relativistic particle beam and plasma modeling. However, a fundamental incompatibility 
between the continuous particle model and the discrete field representation in PIC models causes an aliasing effect. Under certain numerical conditions, the alias modes can interact with the other modes admitted in the E.M. PIC model, causing numerical instabilities that can render unphysical simulation results or even destroy the simulation. Alias modes can also be unstable by themselves, dependent on the deposition scheme employed. We review the numerical dispersion relation of the standard E.M. PIC model based on the Yee FDTD solver to understand the origin of the instabilities.

We abide by the following two guidelines. 1) The eigenmodes in a PIC model consist of spatially continuous, temporally discrete particle eigen-distribution functions, and spatially and temporally discrete eigenfields. The sole cause of aliasing in PIC is the sampling of the continuous spatial variable onto the discrete one. As time and space are independent variables, this spatial aliasing effect will not introduce any temporal aliasing. 2) One should avoid making unnecessary algebraic approximations that may result in an approximation to the original model.

Based on these guidelines, we rigorously derive the faithful numerical dispersion relation for the standard E.M. PIC model with a simple, direct current deposition scheme, which does not exactly conserve electric charge.

We analyze the numerical dispersion of the E.S.-like mode in a 1-D E.M. PIC model with a drifting cold plasma. Accurate numerical solutions and corresponding approximated growth rates are obtained for the finite grid instability. We have shown that in the $\Delta t \rightarrow 0$ limit, and for the parameter regime corresponding to $\Delta t>0$ with $\Delta x \gtrsim \Delta x_{\mathrm{th}}^{\delta}=2 \pi v_{0} \gamma^{3 / 2}$, the dominant finite grid instability is caused by the intersection of the finite grid plasma mode and $q= \pm 1$ alias modes (Figures 3 and 6 ).

On the other hand, for relativistic cold plasma flow under the most relevant simulation parameters, an intersection of the $\omega \approx 0$ mode and alias modes, due to inexact charge conservation, can lead to the most dominant instability (Figure 7). The growth rate for this instability is given by Eq. (54), which shows that there is no threshold grid size or time step size below which this instability will not occur. This instability and associated growth rate have been observed in simulations (Figure 8), which are in excellent agreement with our predictions. An initial investigation of charge conserving schemes in 1-D E.M. codes indicates that each alias mode is unstable by itself, similar to the E.S. case.

Following the guidelines mentioned before allows us to predict the locations of the alias modes in the system and the conditions for numerical instabilities to occur. In future work, we will extend this analysis to include the E.M.-like modes, multiple dimensions, and charge conserving current deposit schemes. This will help to expand the understanding of PIC modeling for relativistic beam and plasma systems.

\section{Acknowledgement}

This work was performed at Los Alamos National Laboratory (LANL) and the University of California Los Angeles (UCLA). Work at LANL was performed under the auspices of the U.S. DOE by Los Alamos National Security, LLC and LANL, and was supported by the LANL Laboratory Directed Research and Development (LDRD) program project 
20130744ECR. Work at UCLA was supported by NSF grant ACI-1339893 and DOE grant DE-NA0001833. We would like to acknowledge useful discussions with Warren Mori, Frank Tsung, Peicheng Yu, Adam Tableman, Zehua Guo, and Gian Luca Delzanno.

\section{References}

[1] C. K. Birdsall and A. B. Langdon, Plasma Physics via Computer Simulation, IOP Publishing Ltd., Bristol, England, 1991.

[2] R. W. Hockney and J. W. Eastwood, Computer Simulation Using Particles, CRC Press, 1988.

[3] J. Dawson, Reviews of Modern Physics 55, 403 (1983).

[4] K. S. YEe, IEEE Transactions on Antennas and Propagation 14, 302 (1966).

[5] A. B. Langdon, Journal of Computational Physics 6, 247 (1970).

[6] J.-L. Vay, C. Geddes, E. Cormier-Michel, and D. Grote, Journal of Computational Physics 230, 5908 (2011).

[7] A. Spitkovsky, The Astrophysical Journal 673, L39 (2008).

[8] S. F. Martins, Pushing the limits of computer simulations for ultra-relativistic scenarios, PhD thesis, Instituto Superior Tecnico, Portugal, 2011.

[9] J.-L. VAY, Physical Review Letters 98, 130405 (2007).

[10] S. F. Martins, R. A. Fonseca, W. Lu, W. B. Mori, and L. O. Silva, Nature Physics 6, 311 (2010).

[11] B. B. Godfrey, Journal of Computational Physics 15, 504 (1974).

[12] B. B. Godfrey, Journal of Computational Physics 19, 58 (1975).

[13] B. B. Godfrey and J.-L. VAy, Journal of Computational Physics 248, 33 (2013).

[14] X. Xu, P. Yu, S. F. Martins, F. S. Tsung, V. K. Decyk, J. Vieira, R. A. Fonseca, W. Lu, L. O. Silva, and W. B. Mori, Computer Physics Communications 184, 2503 (2013).

[15] G. Chen, L. Chacón, and D. Barnes, Journal of Computational Physics 230, 7018 (2011).

[16] J. Villasenor and O. Buneman, Computer Physics Communications 69, 306 (1992).

[17] T. Esirkepov, Computer Physics Communications 135, 144 (2001).

[18] E. Lindman, Journal of Computational Physics 5, 13 (1970). 
[19] A. D. Greenwood, K. L. Cartwright, J. W. Luginsland, and E. A. Baca, Journal of Computational Physics 201, 665 (2004).

[20] C. J. McKinstrie and E. A. Startsev, Physics of Plasmas 2, 3234 (1995).

[21] E. A. R. A. Fonseca, in ICCS, edited by P. M. A. Sloot, volume 2331 of LNCS, pp. 342-351, Springer, 2002.

[22] Roy Gerrit Hemker, Particle-in-cell modeling of plasma-based accelerators in two and three dimensions, PhD thesis, University of California, Los Angeles, 2000. 